OPEN ACCESS

Edited by:

Jiajie Diao,

University of Cincinnati, United States

Reviewed by:

Chuang Wang,

Ningbo University, China

Miou Zhou,

University of California, Los Angeles,

United States

Haijun Tu,

Hunan University, China

*Correspondence:

Juan Chen

chenjuanlinda69@163.com

Anbing Sh

ashi@hust.edu.cn

${ }^{t}$ These authors have contributed equally to this work.

Received: 24 October 2017 Accepted: 04 January 2018

Published: 24 January 2018

Citation:

Shi C, Zeng J, Li Z, Chen Q, Hang W, Xia L, Wu Y, Chen J and

Shi A (2018) Melatonin Mitigates

Kainic Acid-Induced Neuronal Tau

Hyperphosphorylation and Memory

Deficits through Alleviating ER Stress.

Front. Mol. Neurosci. 11:5

doi: 10.3389/fnmol.2018.00005

\section{Melatonin Mitigates Kainic Acid-Induced Neuronal Tau Hyperphosphorylation and Memory Deficits through Alleviating ER Stress}

\author{
Cai Shi' ${ }^{1+}$, Jia Zeng ${ }^{1+}$, Zixi Li ${ }^{2}$, Qingjie Chen ${ }^{1}$, Weijian Hang ${ }^{1}$, Liangtao Xia ${ }^{1}$, Yue Wu ${ }^{1}$, \\ Juan Chen ${ }^{1,3 *}$ and Anbing Shi ${ }^{1,3,4 *}$

\begin{abstract}
${ }^{1}$ Department of Biochemistry and Molecular Biology, School of Basic Medicine and the Collaborative Innovation Center for Brain Science, Tongji Medical College, Huazhong University of Science and Technology, Wuhan, China, ${ }^{2}$ Department of Clinical Laboratory, Tongji Medical College, Huazhong University of Science and Technology, Wuhan, China, ${ }^{3}$ Institute for Brain Research, Huazhong University of Science and Technology, Wuhan, China, ${ }^{4}$ Key Laboratory of Neurological Disease of National Education Ministry, Tongji Medical College, Huazhong University of Science and Technology, Wuhan, China
\end{abstract}

Kainic acid (KA) exposure causes neuronal degeneration featured by Alzheimerlike tau hyperphosphorylation and memory deficits. Melatonin (Mel) is known to protect hippocampal neurons against KA-induced damage. However, the underlying mechanisms remain elusive. In the current study, we investigated the protective effect of melatonin on KA-induced tau hyperphosphorylation by focusing on endoplasmic reticulum (ER) stress-mediated signaling pathways. By using primary hippocampal neurons and mouse brain, we showed that KA treatment specifically induced ER stress and activated GSK-3 $\beta$ and CDK5, two major kinases responsible for tau phosphorylation. Inhibition of ER stress efficiently inactivated GSK-3 $\beta$ and CDK5. Mechanistically, we found that KA-induced ER stress significantly activated calpain, a calcium-dependent protease. Inhibition of ER stress or calpain leads to the reduction in

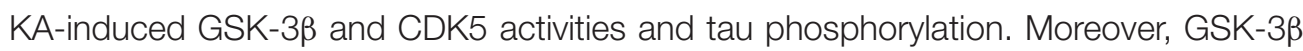
or CDK5 inhibition failed to downregulate ER stress efficiently, suggesting that ER stress functions upstream of GSK-3 $\beta$ or CDK5. Notably, our results revealed that melatonin acts against KA-induced neuronal degeneration and tau hyperphosphorylation via easing ER stress, further highlighting the protective role of melatonin in the KA-induced neuronal defects.

Keywords: melatonin, kainic acid, tau phosphorylation, endoplasmic reticulum stress, calpain

\section{INTRODUCTION}

Microtubule-associated protein tau functions to promote microtubule assembly and maintains the stability of microtubules (Grundke-Iqbal et al., 1986). Hyperphosphorylated tau protein loses its biological activities and leads to the disruption of microtubules, and thus contributes to the neurodegeneration such as Alzheimer's disease (AD) and other tauopathies (Hernandez and Avila, 2008). Neurofibrillary tangles (NFTs) is the primary pathological feature of the AD (Morris et al., 2011). The abnormally hyperphosphorylated tau is the primary component of NFTs, which is 
positively correlated with the decline of memory and cognition in AD patients (Shipton et al., 2011). Therefore, understanding the regulatory mechanisms underlying tau phosphorylation is critical for designing the strategies to arrest AD. Excitatory amino acids have been reported to play a significant role in the pathogenesis of AD (Choi and Rothman, 1990). Kainic acid (KA), an analog of glutamate, has been used to establish excitotoxicity models. Treatment with KA could induce tau hyperphosphorylation and neuronal death through activating GSK-3 $\beta$ and CDK5 pathway in both in vivo and in vitro models (Liang et al., 2009; Tripathi et al., 2010). GSK-3 $\beta$ and CDK5 are two major tau kinases involved in $\mathrm{AD}$-like tau hyperphosphorylation, while the precise intracellular mechanisms through which KA induces tau hyperphosphorylation via GSK-3 $\beta$ and CDK5 activation are still poorly understood.

Endoplasmic reticulum (ER) stress constitutes early events in $\mathrm{AD}$ pathogenesis and has been associated with $\mathrm{AD}$ progression (Salminen et al,, 2009). In the temporal cortex and hippocampus of AD patients, increased level of GRP78 was observed (Hoozemans et al., 2005). The PERK was also found to be abundant in neurons of $\mathrm{AD}$ patients (Hoozemans et al., 2009). The over-activation of glutamate receptors by KA treatment compromises the ER homeostasis and eventually leads to activation of ER stress (Zhang and Zhu, 2011). In addition, ER stress inhibitor salubrinal can attenuate KA-induced hippocampal cell death (Kim et al., 2014), suggesting that KA is capable of inducing ER stress.

Melatonin (Mel), a tryptophan metabolite, is synthesized by the pineal gland and many other organs (Acuna-Castroviejo et al., 2014). Melatonin is well known for its roles in the regulation of circadian rhythms, sleep, mood, reproduction, tumor growth, and aging (Wu and Swaab, 2005). Deficiency of melatonin in the cerebrospinal fluid (CSF) and blood is highly correlated with the progression of cognitive impairment (Liu et al., 1999). For patients suffering from the very early stage of the $\mathrm{AD}$, the decline of melatonin in CSF and blood serve as a sensitive indicator of cognitive dysfunction (Zhou et al., 2003). Importantly, melatonin supplementation can effectively slow down the progress of hippocampus neuronal degeneration and improve the cognition ability (Brusco et al., 2000). Furthermore, melatonin was reported to regulate the activities of CDK5 and GSK-3 $\beta$ in hippocampal neurons (Peng et al., 2013). Accumulating evidence suggests that the neuroprotective effect of melatonin is associated with the modulation of ER stress. For instance, melatonin was used to alleviate ER stress in a rat model of renal warm ischemia reperfusion (Hadj Ayed Tka et al., 2015). In murine splenocytes, melatonin reversed ER stressmediated apoptosis induced by the herbicide atrazine (Sharma et al., 2014). Constant light illumination increased expression of GRP78 in rats, supplement with melatonin could efficiently protect the cells from the ER stress-induced impairments (Ling et al., 2009). Consistently, in the current study, we show that KA affects CDK5/GSK-3 $\beta$ and tau phosphorylation via ER stress induction. Melatonin executes protective effects against KA-induced neuronal excitotoxicity through ER stress alleviation.

\section{MATERIALS AND METHODS}

\section{Cell Culture and Treatments}

Primary hippocampal neurons were prepared from 2-dayold Sprague-Dawley rats using the described protocols, with modifications (Isaev et al., 2002). The tissue was briefly digested with $0.25 \%$ trypsin in phosphate-buffered saline (PBS) for $20 \mathrm{~min}$ at $37^{\circ} \mathrm{C}$ followed by mechanical dissociation. Primary hippocampal neurons were seeded in poly-L-lysine-coated plates $\left(120,000\right.$ cells $\left./ \mathrm{cm}^{2}\right)$ and grown in neurobasal medium with B-27 serum-free supplement (Gibco, Grand Island, NY, United States), $100 \mathrm{U} / \mathrm{mL}$ penicillin, $100 \mathrm{~g} / \mathrm{mL}$ streptomycin, and $2 \mathrm{mM}$ L-glutamine. The cultures were maintained in a humid incubator aerated with $95 \%$ air and $5 \% \mathrm{CO}_{2}$ at $37^{\circ} \mathrm{C}$. The medium was changed starting from the 4 th day by replacing half of the medium twice a week. Serum-free primary neuron cultures were utilized for the experiments after 8 days.

In experiment 1 , the primary hippocampal neurons were pre-treated with or without melatonin $(50 \mu \mathrm{M})$ for $1 \mathrm{~h}$ and then stimulated with $\mathrm{KA}(50 \mu \mathrm{M})$ or thapsigargin (TG, $2 \mu \mathrm{M}$, Sigma, St. Louis, MO, United States) for $8 \mathrm{~h}$. Melatonin (Sigma, St. Louis, MO, United States) was first dissolved in absolute ethanol at a concentration of $50 \mathrm{mM}$ and diluted with culture medium to the final concentration. KA (Abcam, Cambridge, MA, United States) was first dissolved in dimethyl sulfoxide (DMSO) at a concentration of $100 \mathrm{mM}$ and then diluted with culture medium to the final concentration. TG was dissolved in DMSO at a concentration of $1 \mathrm{mM}$ as stock solutions and then diluted with medium to $2 \mu \mathrm{M}$. Corresponding dilutions of ethanol and DMSO were given to the control group.

In experiment 2, the primary hippocampal neurons were pre-treated with or without Salubrinal (Sal, an ER stress inhibitor, 5 or $10 \mu \mathrm{M}$, Sigma, St. Louis, MO, United States), SB216763 (SB, a specific GSK-3 $\beta$ inhibitor, 2 or $20 \mu \mathrm{M}$, Tocris Bioscience, Bristol, United Kingdom), roscovitine (Ros, a CDK5 inhibitor, 50 or $100 \mu \mathrm{M}$, Sigma, St. Louis, MO, United States) and MDL28170 (MDL, a calpain inhibitor, $10 \mu \mathrm{M}$, Sigma, St. Louis, MO, United States) for $1 \mathrm{~h}$, and then stimulated with KA $(50 \mu \mathrm{M})$ for $8 \mathrm{~h}$. Sal, SB, and Ros were dissolved in DMSO as stock solutions at the concentration of $20 \mathrm{mM}$, then further diluted into cell culture medium to a final concentration as described before. A stock solution of the MDL was prepared in DMSO at the concentration of $5 \mathrm{mM}$ then further diluted into cell culture medium to the final concentration of $10 \mu \mathrm{M}$. Corresponding dilutions of DMSO were given to control, and KA-treated group.

\section{Animals and Treatments}

Adult male C57BL/6 mice, weighing $25 \pm 2$ g, were supplied by the Experimental Animal Center of Tongji Medical College. All experimental procedures were approved by the Animal Care and Use Committee at the Huazhong University of Science and Technology and were performed in compliance with National Institutes of Health guidelines on the ethical use of animals. The mice were housed five per cage in a room maintained at $22 \pm 2^{\circ} \mathrm{C}$ 
with an alternating 12-h light-dark cycle. Food and water were available ad libitum.

In experiment 1 , based on the previous study, a total of 42 mice were treated with an intraperitoneal (i.p.) injection of $30 \mathrm{mg} / \mathrm{kg} \mathrm{KA}$ (Abcam, Cambridge, MA, United States) emulsified in $0.9 \%$ normal saline (Crespo-Biel et al., 2010). To validate the neurotoxic induction of KA, behavioral observations were made every $30 \mathrm{~min}$ for $4 \mathrm{~h}$ after KA injection. The mice which did not display general limbic seizure activity within $90 \mathrm{~min}$ after the KA injection were excluded from further study. Thus, the final numbers of KA groups were 35. Mice in the control group $(n=7)$ were injected with saline. Animals in the experimental group were sacrificed 6 and $12 \mathrm{~h}$ and 1,3 , and 7 days after KA treatment.

In experiment 2, a total of 36 mice were divided randomly into three groups: KA-only group (KA), melatonin (Sigma, St. Louis, MO, United States) administration before KA group $(\mathrm{Mel}+\mathrm{KA})$, and vehicle-treated control group (Con). Based on the previous study (Jain et al., 2013), melatonin was dissolved in absolute ethanol and diluted in saline to a final concentration before injection. The mice in the Mel+KA group were given i.p. injections of $20 \mathrm{mg} / \mathrm{kg}$ melatonin once $30 \mathrm{~min}$ before the injection of KA on the 1st day and a single dose per day for a total of 3 days (Jain et al., 2013). The mice in each group were sacrificed on the 4th day after KA treatment. All mice were sacrificed after KA treatment, and the hippocampal tissue was harvested for further tests.

\section{Morris Water Maze Test}

To assess the cognitive changes, Morris water maze (MWM) test was conducted in a circular water tank $(120 \mathrm{~cm}$ in diameter and $35 \mathrm{~cm}$ in height) filled with water to the depth of $21 \mathrm{~cm}$ and maintained at $25 \pm 1{ }^{\circ} \mathrm{C}$. The tank was divided into four equal quadrants. A submerged square platform $(15 \mathrm{~cm}$ in width and $20 \mathrm{~cm}$ in height) was placed in the third quadrant of the tank with its top surface $1 \mathrm{~cm}$ below the water surface. The mice were placed in the pool at four possible start locations facing the wall of the pool, and a camera was simultaneously activated. Each mouse was allowed up to $60 \mathrm{~s}$ to locate the platform. The trial was terminated when the mouse found the platform within $60 \mathrm{~s}$. If a mouse failed to find the platform within $60 \mathrm{~s}$, it was guided by a researcher to locate the platform and allowed to stay for 2-3 s. Each mouse was conditioned three times per day for 2 days to let them adapt to the pool environment (visible platform training) and then tested three times per day for 6 days to find the hidden platform (hidden platform training). The latency (the time taken to locate the platform in water), distance, and swim speed were recorded using an automated video tracking software package (NoldusEtho Vision 2.3.19, Netherlands). For data analysis, the pool was divided into four quadrants. During the visible platform training, the platform was moved to a different quadrant for each session. During the hidden platform training, the platform location was identical to each mouse. Behaviors of the mice were tracked using EthoVision 3.0, and the escape latency was analyzed. In each trial, the average latency time for each mouse was calculated and recorded.

\section{Western Blotting}

Total proteins were extracted by using a protein extraction kit (Pierce, IL, United States) following the manufacturer's protocol. Protein extracts were dissolved in $10-15 \%$ sodium dodecyl sulfate polyacrylamide gel and then transferred to a nitrocellulose membrane at $150 \mathrm{~mA}$. After being blocked with 5\% non-fat skimmed milk [diluted with Tris-buffered saline containing $0.1 \%$ Tween 20 (TBST)] for $1 \mathrm{~h}$ at room temperature, the membrane containing the protein extracts was incubated overnight with primary antibody (diluted with $2 \%$ bovine serum albumin in TBST) at $4^{\circ} \mathrm{C}$. The following primary antibodies were used: anti- $\beta$-actin (1:3000, Santa Cruz, CA, United States), anti-tau/ps396 (1:1000, Abcam, Cambridge, MA, United States), anti-tau/ps199 (1:1000, Abcam, Cambridge, MA, United States), and anti-tau-5 (1:1000, Abcam, Cambridge, MA, United States); anti-CDK5 (1:1000, Santa Cruz, CA, United States); antiGSK-3 $\beta$ (1:1000, CST, Framingham, MA, United States); anti-calpain (1:1000, Santa Cruz, CA, United States); and anti-p35 (1:1000, Santa Cruz, CA, United States), anti-GRP78 (1:2000, Abcam, Cambridge, MA, United States); anti-ATF-6 (1:1500, Abcam, Cambridge, MA, United States); anti-ATF-4 (1:1000, Abcam, Cambridge, MA, United States); anti-IRE-1 (1:1500, Abcam, Cambridge, MA, United States); anti-eIF-2 $\alpha$ (1:1000, CST, Framingham, MA, United States) and anti-pPERK (1:500, Santa Cruz, CA, United States). On the 2nd day, proteins were visualized using the enhanced chemiluminescence detection system (Pierce, IL, United States) after incubating with corresponding secondary antibodies (1:1000, Amersham Pharmacia Biotech, Buckinghamshire, United Kingdom) and then exposed to medical x-ray film. For the quantitative analysis of band intensity, we calculated the band intensity ratio for normalization by using a gel image analyzer (JS380; Peiqing Science and Technology, Shanghai, China). For each blot, multiply the background subtracted density of the target protein in each lane by the ratio of the density of the loading control (such as housekeeping protein) from a control sample of all the study blots to the other lanes in the gel. This will give the normalized density to the loading control (NDL). Calculate the fold difference for each replicate by dividing NDL from each lane by the NDL from the control sample.

\section{Immunochemistry}

Mouse hippocampal tissues were frozen and sectioned consecutively at $10 \mu \mathrm{m}$, with three sections for each embedded sample. The sections were incubated overnight at $4^{\circ} \mathrm{C}$ with tau ps-396 antibody (1:1000), respectively. After incubation with horseradish peroxidase-conjugated secondary antibody (Jackson Immuno Research, West Grove, PA, United States) for $2 \mathrm{~h}$ at room temperature, sections were washed and developed with $0.05 \%$ diaminobenzidine (DAB) plus $0.015 \%$ hydrogen peroxide in PBS. The DAB-stained sections were air-dried, counterstained with Mayer's hematoxylin, dehydrated, cleared, and coverslipped. Finally, the sections were visualized with DAB for light microscopy examination. For every section, DG, CA1, and CA3 areas of the hippocampus were examined with an Olympus AX-70 microscope equipped with a motorized stage (Olympus, NY, United States). Images were captured 

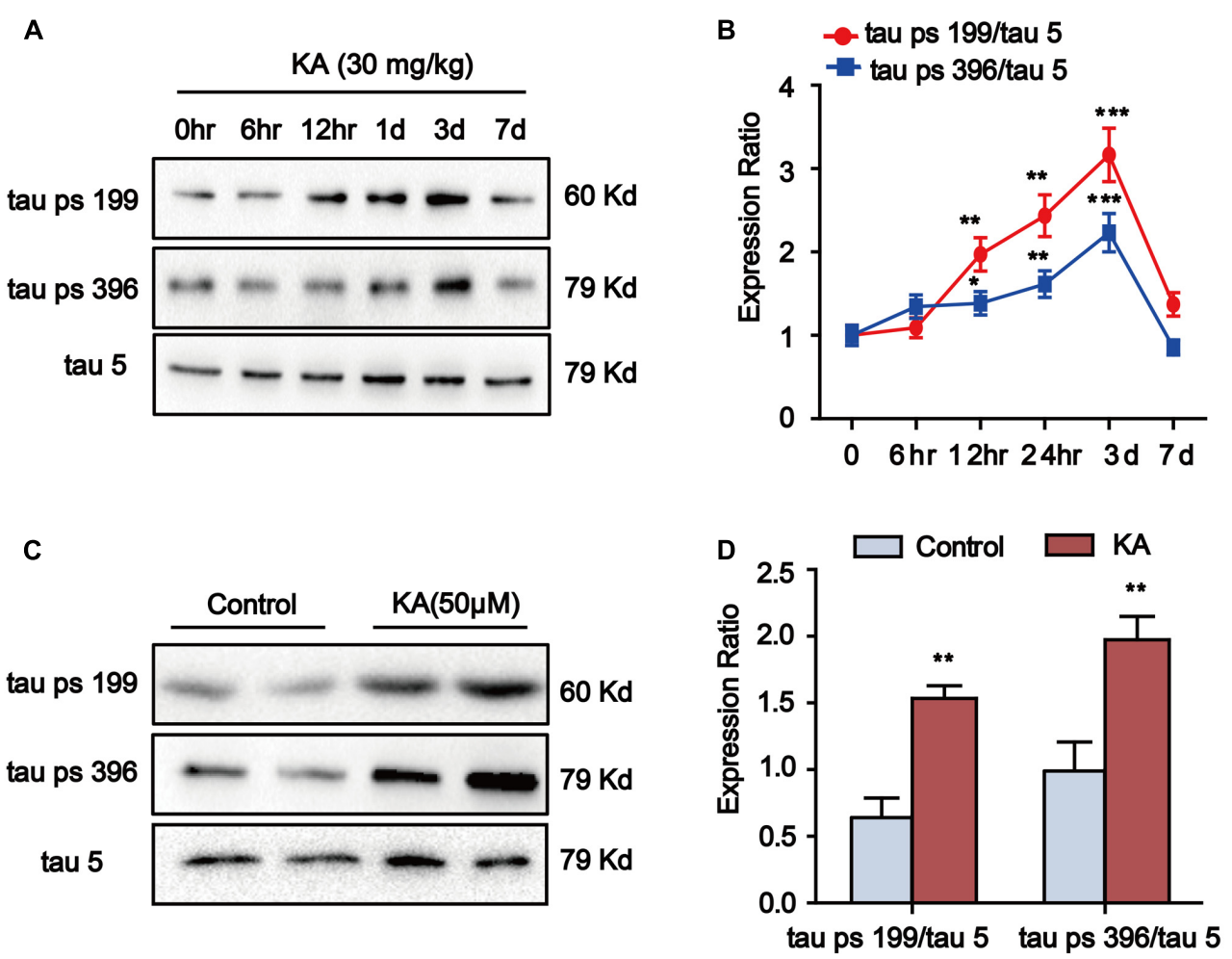

FIGURE 1 | Kainic acid (KA) augments tau phosphorylation level in vivo and in vitro. (A,B) Phosphorylation levels of tau (Ser199 and Ser396) in KA-treated mouse hippocampus at different time points. (C,D) Phosphorylation levels of tau (Ser199 and Ser396) in KA-treated primary hippocampal neurons. $(* * P<0.01$,

*** $P<0.001$ vs. controls; the significant difference from the respective values were determined by one-way analysis of variance test. $N=3$ for western blotting).

at $20 \times$ magnification. Image analysis of all sections was performed within 4 weeks after immunohistochemistry with the tau ps-396 antibody. For quantification, we used the Fiji/ImageJ open source software to perform digital slide image processing. To overcome potential heterogeneity in intensities across different in-slide areas, we calculated the mean reciprocal intensity (RI) of a region of interest (ROI). A uniformly sized ROI was placed over the mossy fibers staining using the 'Draw' tool, and a standard RBG (red-green-blue) image was produced from each respective slide, by applying DAB-specific color deconvolution. Mean intensity was calculated using the 'Measure' function under the 'Analyze' menu of the Fiji software. Since the maximum intensity value of an RGB image analyzed in Fiji is 250, we subtracted the mean intensity of each stained ROI from 250, thereby deriving a RI that was directly proportional to the amount of chromogen present.

\section{Enzyme Activity Assay}

The calpain activity was measured using Calpain Substrate II Kit (Merck Millipore, Darmstadt, Germany). Cells were washed twice with PBS and lysed on ice with extraction buffer. The total protein extracted $(50 \mu \mathrm{g})$ was incubated with $150 \mu \mathrm{L}$ substrate and $1 \mathrm{~mL}$ reaction buffer for $100 \mathrm{~min}$ at $37^{\circ} \mathrm{C}$. The release of 7-amino-4-methyl-coumarin (AMC) from the reaction was monitored at $440 \mathrm{~nm}$ of emission using a fluorescence spectrometer. Fluorescence units were converted into AMC release using standard curve. The activity of calpain was expressed and documented in pmol of AMC cleaved per minute per milligram of protein.

GSK-3 activity was assessed using $\gamma^{-32} \mathrm{P}$-ATP assay. The reaction mixture contained $30 \mathrm{mM}$ Tris- $\mathrm{HCl}$ ( $\mathrm{pH} 7.4), 10 \mathrm{mM}$ $\mathrm{MgCl}_{2}, 10 \mathrm{mM} \mathrm{NaF}, 1 \mathrm{mM} \mathrm{Na} \mathrm{VO}_{4}, 2 \mathrm{mM}$ EGTA, $0.2 \mathrm{mM}$ ATP, $2000 \mathrm{cpm} / \mathrm{pmol} \gamma$ - ${ }^{32} \mathrm{P}$-ATP, $10 \mathrm{mM} \beta$-mercaptoethanol, and $7.5 \mu \mathrm{g}$ protein sample. After incubating for $30 \mathrm{~min}$ at $30^{\circ} \mathrm{C}$, the reaction was terminated by adding $300 \mathrm{mmol} / \mathrm{L}$ $\mathrm{H}_{3} \mathrm{PO}_{4}$. The $\gamma_{-}{ }^{32} \mathrm{P}$-labeled peptide was blotted on a phosphorcellulose paper, then washed with $75 \mathrm{mmol} / \mathrm{L} \mathrm{H}_{3} \mathrm{PO}_{4}$ three times and dried at $60^{\circ} \mathrm{C}$ overnight. Incorporation of $\gamma^{-32} \mathrm{P}$ was quantified in counts per minute (cpm) using a scintillation counter.

CDK-5 activity was assayed using the procedure described by Pigino et al. (1997). A sample containing $200 \mu \mathrm{g}$ total cellular protein was used for immunoprecipitation with the anti-CDK-5 antibody (1:50) and Protein G Plus-Agarose (Santa Cruz, CA, United States). The immunoprecipitates were rinsed three times with lysis buffer and once with kinase buffer (50 mM HEPES, $\mathrm{pH} 7.0,10 \mathrm{mM} \mathrm{MgCl} 2,1 \mathrm{mM} \mathrm{DTT}$, and $1 \mathrm{mM}$ cold ATP). The rinsed agarose beads were incubated with kinase buffer containing $2.5 \mu \mathrm{g}$ histone $\mathrm{H} 1$ plus $200 \mu \mathrm{M} \gamma-{ }^{32} \mathrm{P}$-ATP in a final volume of $50 \mu \mathrm{L}$ for $30 \mathrm{~min}$ at $30^{\circ} \mathrm{C}$, and the reaction products were determined using a scintillation counter. 
A
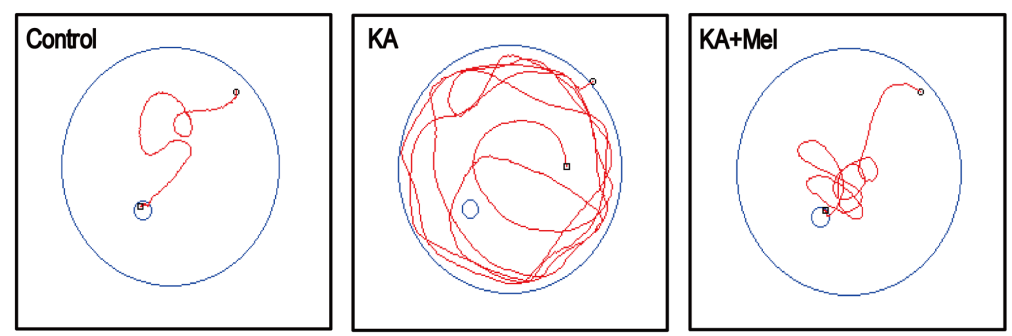

B
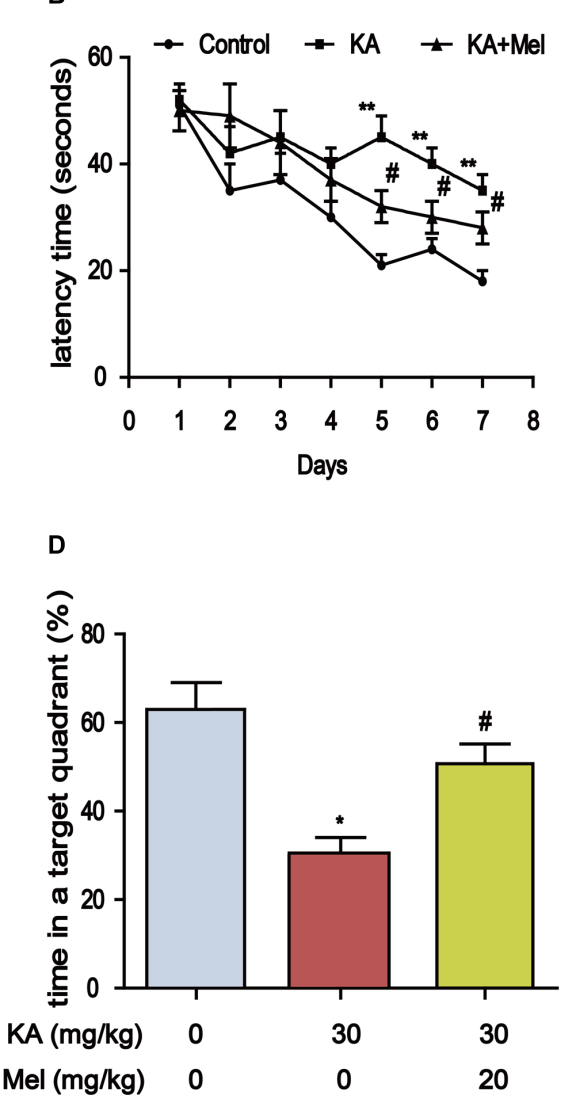

C
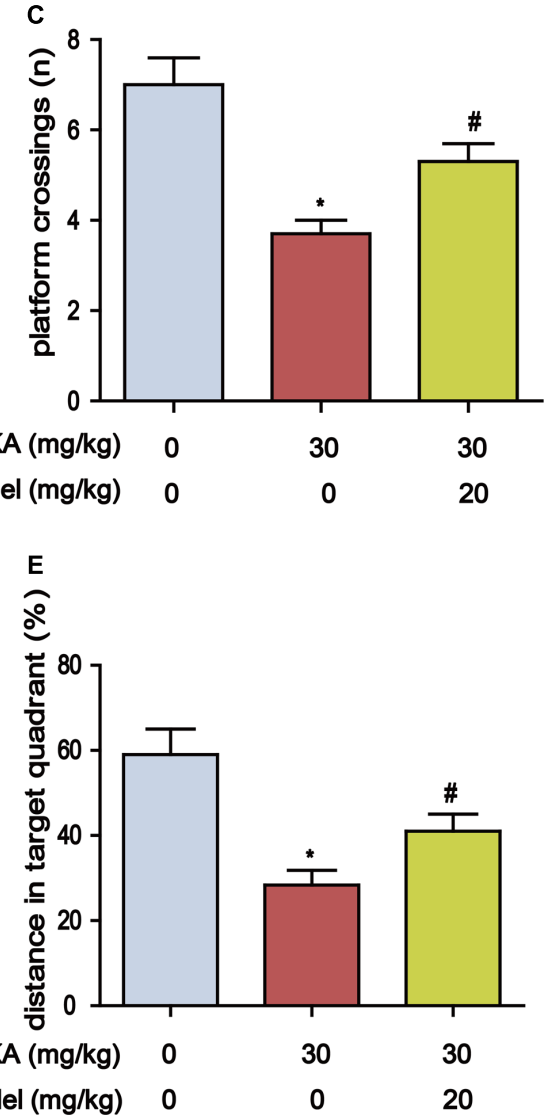

FIGURE 2 | Melatonin mitigates KA-induced memory deficits by using Morris Water Maze test. (A) The trajectory of rats in MWM. (B) Escape latency analysis in the training trials. (C) The frequency of crossing over the former location during the probe trial. (D) The percentage of time spent in target quadrant during the probe trial. (E) The percentage of distance in the target quadrant during the probe trial. ( ${ }^{*} P<0.05,{ }^{* *} P<0.01$ vs. the control group; ${ }^{\#} P<0.05$ vs. the KA-only group, $n=7$ per group for MWM test; the significant difference from the respective values were determined by two-way analysis of variance test).

\section{Measurement of Malondialdehyde and Superoxide Dismutase}

Cells were seeded in 6-well culture plates. After $8 \mathrm{~h}$ of treatment with $\mathrm{KA}$ or Mel+KA, cells were washed twice with PBS (pH 7.4, $4^{\circ} \mathrm{C}$ ) and lysed with buffer containing $10 \mathrm{mM}$ Tris- $\mathrm{HCl}(\mathrm{pH} 7.4)$, $10 \mathrm{mM}$ ethylenediaminetetra-acetic acid and $0.2 \%$ Triton X-100 on ice. The cells were scraped from the wells and centrifuged at $17000 \times g$ for $15 \mathrm{~min}$ at $4^{\circ} \mathrm{C}$. Superoxide dismutase activity in the supernatant was determined by assessing the inhibition of pyrogallolautoxidation (Marklund and Marklund, 1974).

Levels of Malondialdehyde (MDA), a metabolite of lipid peroxides, were estimated as reactive substances by thiobarbituric acid addition (Kobe et al., 2002). Primary hippocampal neurons were washed twice and collected in cold PBS and centrifuged at $3000 \times g$ for $5 \mathrm{~min}$. Then, the pellets were thoroughly mixed with $4 \mathrm{~mL}$ of $0.083 \mathrm{M}$ sulfuric acid and $0.5 \mathrm{~mL}$ of $10 \%$ phosphotungstic acid. After centrifugation at $3000 \times g$ for $10 \mathrm{~min}$, the liquid phase was decanted. Four milliliters of double-distilled water and thiobarbituric acid reagent $(0.67 \%$ 2-thiobarbituric acid/acetic acid 1:1) were added to each sample, mixed, and heated in a water bath at $95^{\circ} \mathrm{C}$ for $1 \mathrm{~h}$. The samples were cooled with tap water, $5 \mathrm{~mL}$ of N-butanol-alcohol was added. The samples were vigorously shaken for $1 \mathrm{~min}$ and centrifuged. The N-butanol-alcohol phase, which contains the lipid peroxides, was used for MDA analysis with a 
A

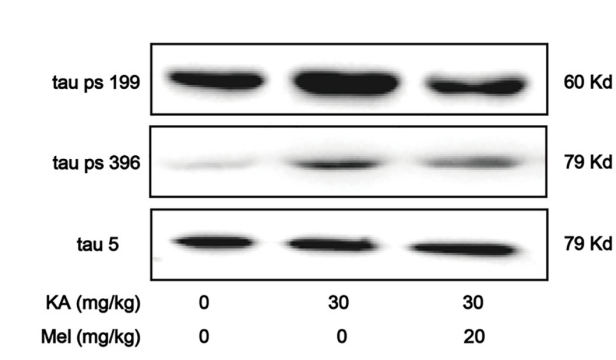

B

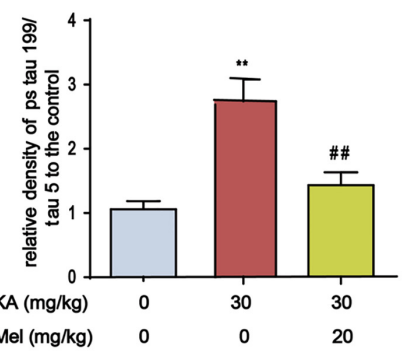

C

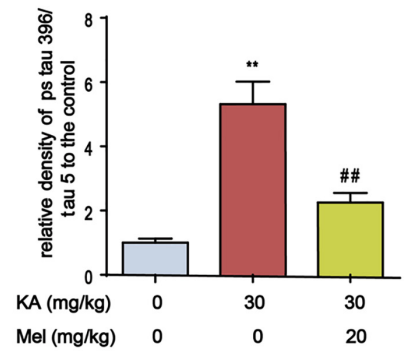

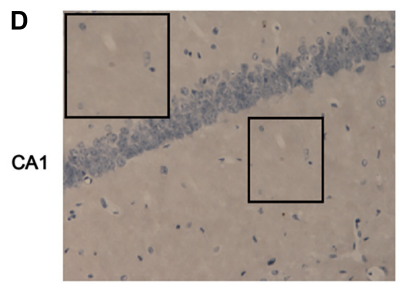
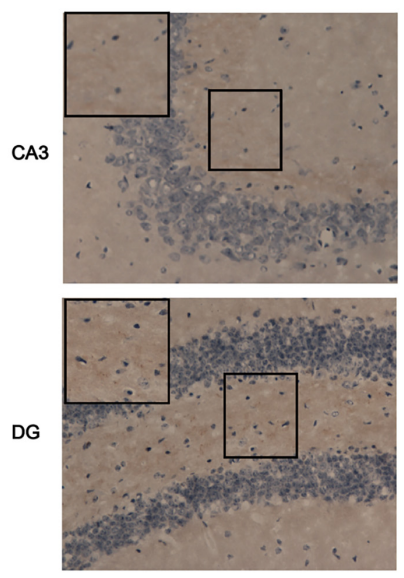

Control
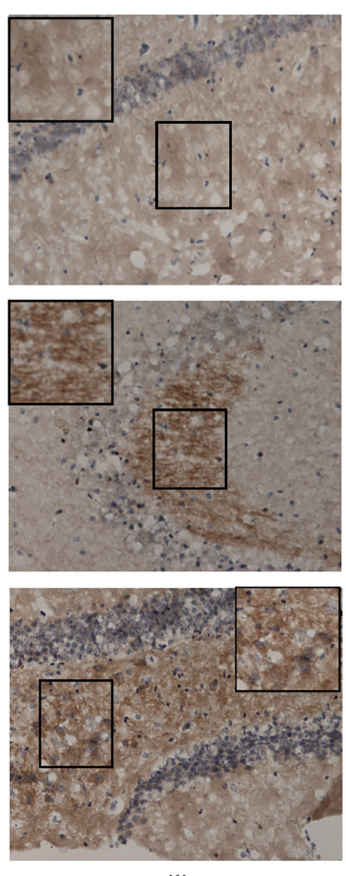

KA
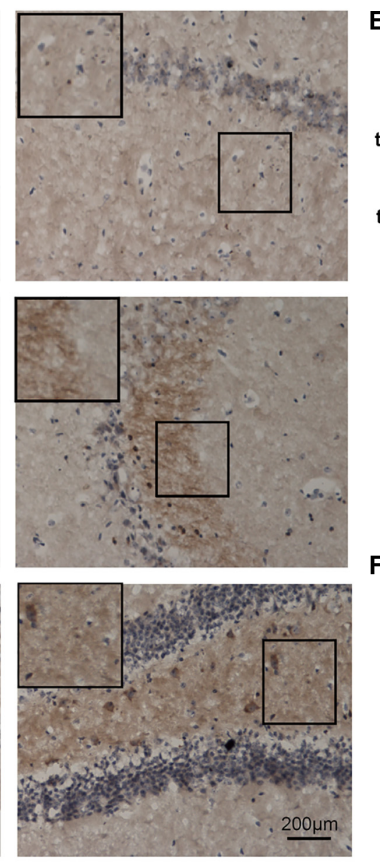

$\mathrm{KA}+\mathrm{Mel}$

E
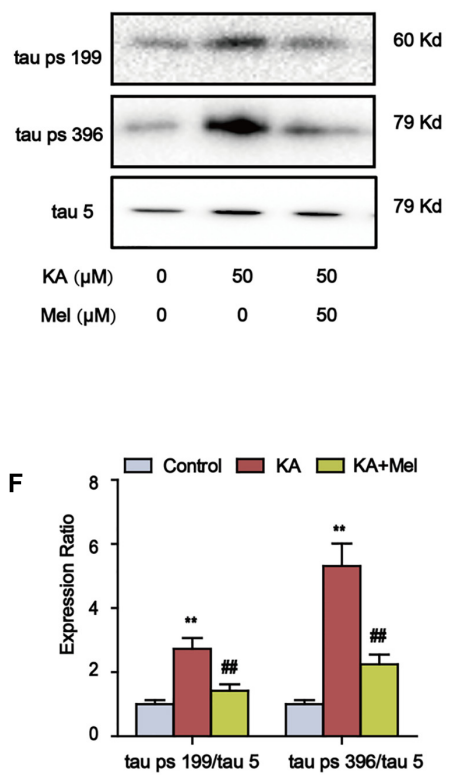

FIGURE 3 | Melatonin ameliorates KA-induced tau hyperphosphorylation. (A-C) Phosphorylation levels of tau (Ser199 and Ser396) in KA and/or Mel-treated mouse hippocampus. The KA group mice were given were given i.p. injection of $30 \mathrm{mg} / \mathrm{kg} \mathrm{KA}$. The Mel+KA group mice were given i.p. injections of $20 \mathrm{mg} / \mathrm{kg}$ melatonin once $30 \mathrm{~min}$ before the injection of $30 \mathrm{mg} / \mathrm{kg} \mathrm{KA}$ on the 1 st day and a single dose per day for a total of 3 days. (D) Immunohistochemical staining of tau phosphorylation at Ser396 in KA and/or Mel-treated mouse hippocampus. (E,F) Phosphorylation levels of tau (Ser199 and Ser396) in KA and/or Mel-treated primary hippocampal neurons. The primary hippocampal neurons were pre-treated with or without melatonin (50 $\mu \mathrm{M})$ for $1 \mathrm{~h}$ and then stimulated with $\mathrm{KA}$ ( $50 \mathrm{\mu M}$ ) for $8 \mathrm{~h}$. (**P $<0.01$ vs. the control group; ${ }^{\# \# ~} P<0.01$ vs. the KA-only group, $n=7$ per group for immunohistochemical staining test; $n=3$ for western blotting; the significant difference from the respective values were determined by one-way analysis of variance test).

fluorospectrophotometer (F-2000, Hitachi Ltd., Tokyo, Japan) using excitation/emission wavelengths of 515/553 nm. Freshly diluted tetramethoxypropane, which yields MDA, was used as a standard, and the results are expressed as nanomoles of MDA equivalents. The protein concentration was measured using the BCA reagent (Pierce, FL, United States).

\section{Flow Cytometric Measurement of Cytosolic $\mathrm{Ca}^{2+}$ Concentration $\left(\left[\mathrm{Ca}^{2+}\right] \mathrm{c}\right)$ and Endoplasmic Reticulum $\mathrm{Ca}^{2+}$ Levels $\left(\left[\mathrm{Ca}^{2+}\right]_{\mathrm{ER}}\right)$}

Intracellular $\mathrm{Ca}^{2+}$ concentration was measured using the calcium indicator Fura-2 AM. After treatment, primary hippocampal neurons grown on glass slides were loaded with $5 \mu \mathrm{M}$ Fura-2 AM for $45 \mathrm{~min}$ in Hanks Balanced Salt Solution (HBSS), and equilibrated for $30 \mathrm{~min}$ in the dark at room temperature. Cells were then placed in an open-bath imaging chamber containing HBSS. Using a Nikon inverted epifluorescence microscope, neurons were excited at 345 and $385 \mathrm{~nm}$ and the emission fluorescence at $510 \mathrm{~nm}$ was recorded. Images were collected and analyzed with the MetaFluor image-processing software, and the results were calculated and reported as $F_{\mathrm{t}} / F_{0}$ and area under curve (AUC).

It has been verified that Mag-Fluo-4-AM was selectively labeled on ER, making its specificity for measuring $\left[\mathrm{Ca}^{2+}\right]_{\mathrm{ER}}$ (Wang et al., 2015). After corresponding treatment, cells were incubated with $2 \mu \mathrm{M}$ Mag-Fluo-4-AM and 0.02\% (w/v) Pluronic 
A

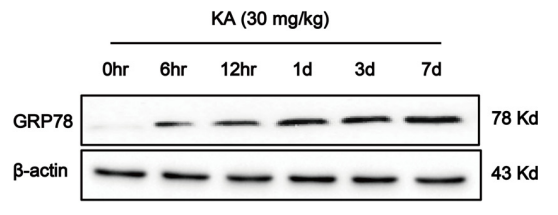

C

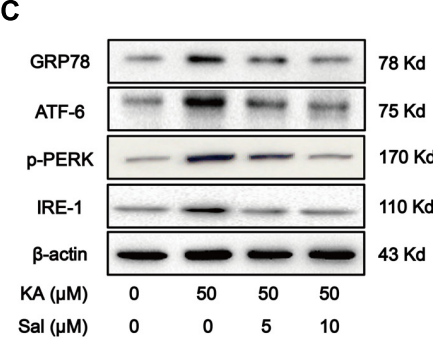

D
B

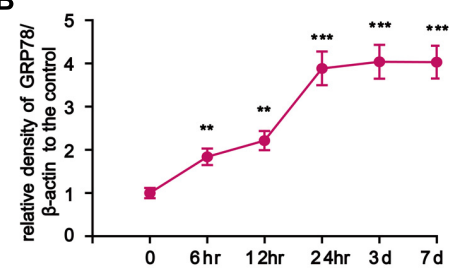

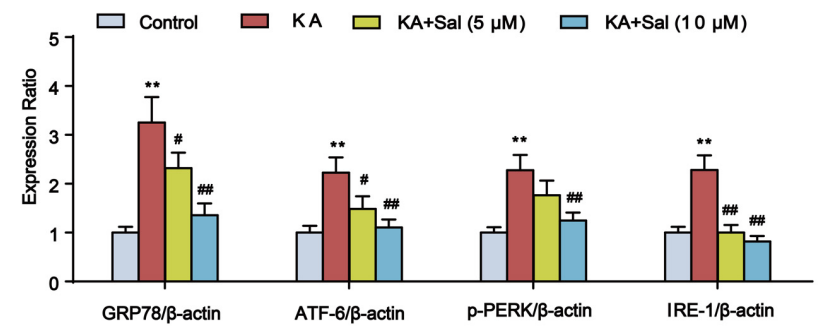

E
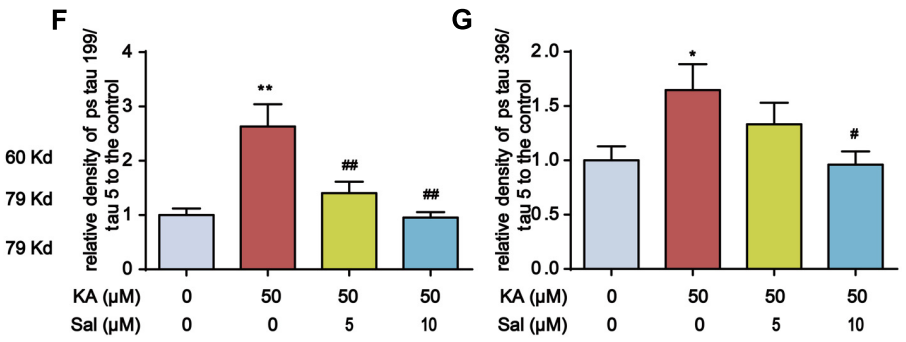

$\mathbf{G}$

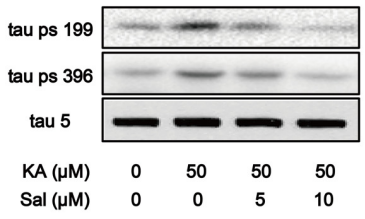

FIGURE 4 | Kainic acid-induced tau hyperphosphorylation is highly correlated with ER stress. (A,B) Protein levels of GRP78 in KA-treated mouse hippocampus at different time points. (C,D) Protein levels of GRP78, ATF-6, p-PERK, and IRE-1 in KA and/or Sal-treated primary hippocampal neurons. The primary hippocampal neurons were pre-treated with or without Salubrinal (Sal, an ER stress inhibitor, 5 or $10 \mu \mathrm{M}$ ) for $1 \mathrm{~h}$ and then stimulated with $\mathrm{KA}$ ( $50 \mu \mathrm{M}$ ) for $8 \mathrm{~h}$.

(E-G) Phosphorylation levels of tau (Ser199 and Ser396) in KA and/or Sal-treated primary hippocampal neurons. $(* P<0.05, * * P<0.01, * * * P<0.001$ vs. controls; ${ }^{\#} P<0.05$; ${ }^{\#} P<0.01$ vs. the KA group; the significant difference from the respective values were determined by one-way analysis of variance test. $N=3$ for western blotting).

F-127 for $30 \mathrm{~min}$ at $37^{\circ} \mathrm{C}$ in dark. $488 \mathrm{~nm}$ laser was used to excite Mag-Fluo-4 fluorescence and the emission fluorescence at $510 \mathrm{~nm}$ was recorded. Images were collected and analyzed with the MetaFluor image-processing software.

\section{Statistical Analysis}

Data were expressed as the mean \pm standard deviation and analyzed using SPSS 10.0 statistical software (SPSS Inc., Chicago, IL, United States). The one-way and two-way ANOVA tests were used to determine the significance of differences among groups $(P<0.05, P<0.01, P<0.001)$.

\section{RESULTS}

\section{Kainic Acid Treatment Boosts Tau Phosphorylation in Hippocampal Neurons}

Kainic acid (KA) has been known to cause status epileptics, neurodegeneration and memory loss (Milatovic et al., 2001). To validate the neuronal toxicity of $\mathrm{KA}$, we treated the mouse with i.p. injections (30 mg/kg KA; 6 and $12 \mathrm{~h}$ and 1, 3, and 7 days) and measured the hippocampal phosphorylation level of tau protein. In the groups of 12-h, 1- and 3-day after KA treatment, we observed the significant increase in tau phosphorylation at Ser199 and Ser396 in the hippocampus. Seven days after KA administration, there were no further changes in tau phosphorylation (Figures 1A,B). Thus, we harvested hippocampus tissue 3 days after KA treatment for the experiments.

We further assayed the tau phosphorylation in primary hippocampal neurons. Eight hours after KA treatment, we noticed that the tau phosphorylation at Ser199 and Ser396 sites significantly increased in primary hippocampal neurons and the level of tau phosphorylation reached the highest point at 16-h (Figures 1C,D). Considering the efficiency of melatonin treatment (Xue et al., 2017), we chose to use the 8-h time point in our assays. It is worth noting that the phosphorylation level of tau started to increase after $12 \mathrm{~h}$ of KA treatment and reached the peak level at 3-day in the animal model (Figures 1A,B). We speculate that the tau phosphorylation boost latency is due to the late onset time of KA in the live animals. Taken together, our observations indicated that KA treatment could efficiently boost tau phosphorylation both in vivo and in vitro. 

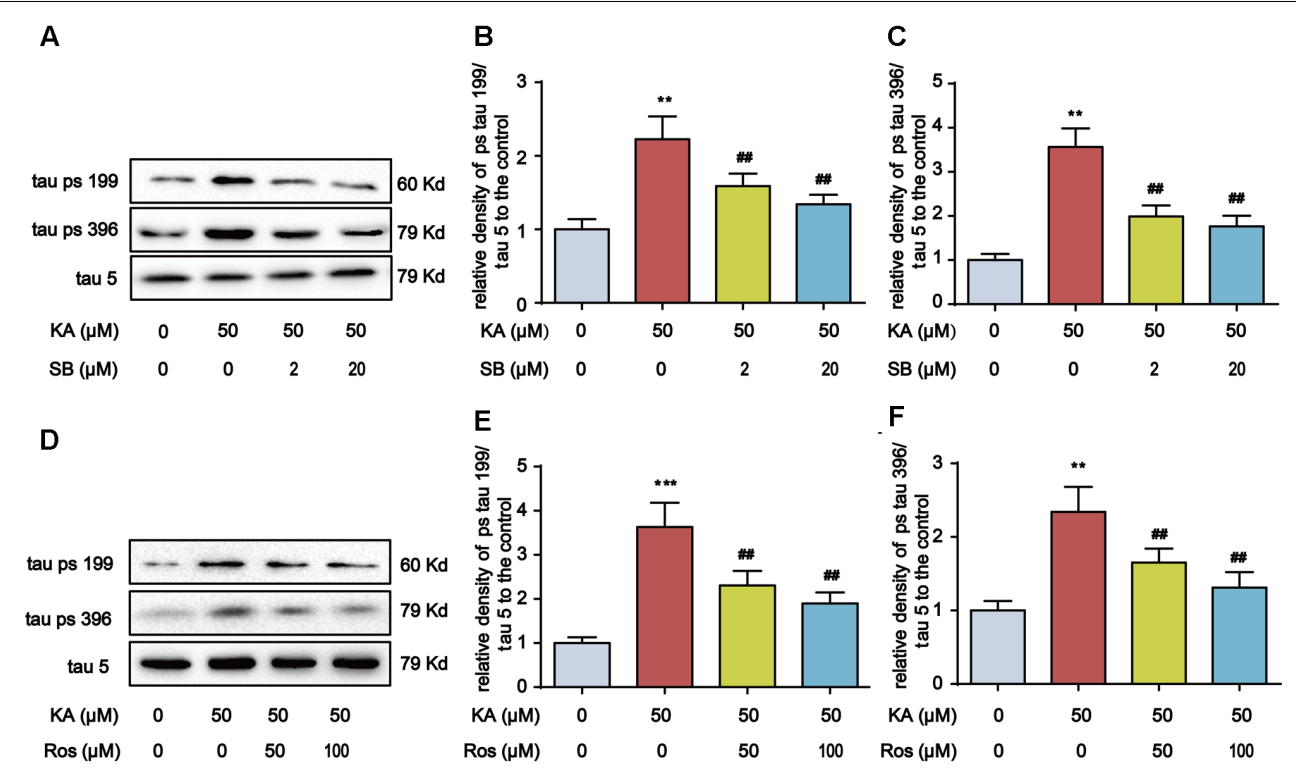

FIGURE 5 | SB216763 (SB)/Roscovitine (Ros) inhibits tau hyperphosphorylation in primary hippocampal neurons. (A-C) Phosphorylation levels of tau (Ser199 and Ser396) in KA and/or SB-treated primary hippocampal neurons. The primary hippocampal neurons were pre-treated with or without SB216763 (SB, a specific GSK-3 3 inhibitor, 2 or $20 \mu \mathrm{M}$ ) for $1 \mathrm{~h}$ and then stimulated with KA (50 $\mu \mathrm{M}$ ) for $8 \mathrm{~h}$. (D-F) Phosphorylation levels of tau (Ser199 and Ser396) in KA and/or Ros-treated primary hippocampal neurons. The primary hippocampal neurons were pre-treated with or without roscovitine (Ros, a CDK5 inhibitor, 50 or $100 \mu \mathrm{M}$ ) for $1 \mathrm{~h}$ and then stimulated with $\mathrm{KA}(50 \mu \mathrm{M})$ for $8 \mathrm{~h}$. ${ }^{* *} P<0.01,{ }^{* * *} P<0.001 \mathrm{vs}$. controls; ${ }^{\# \#} P<0.01 \mathrm{vs}$. KA group; the significant difference from the respective values were determined by one-way analysis of variance test. $N=3$ for western blotting).

\section{Melatonin Mitigates Kainic Acid-Induced Memory Deficits and Tau Hyperphosphorylation}

Deficiency of melatonin in the CSF and blood is highly correlated with the progression of cognitive impairment (Liu et al., 1999). Melatonin supplementation slows down neuronal degeneration progression in rat hippocampus and improves cognition ability (Zhou et al., 2003). To assess whether melatonin could alleviate KA-induced memory impairment, we performed MWM test to assay the learning ability of the mouse via training with a hidden platform. We observed that the mean escape latency in KA-only group $(\mathrm{F} 6,84=15.16, P<0.001)$ increased which compared to control group, and the escape latency of Mel+KA group was shorter than that of KA-only group (F6, $84=9.214$, $P<0.001$ ) (Figures 2A,B). On the 7th day, we removed the platform and conducted the probe trial. The platform crossing frequency of Mel+KA group was higher than those of KA-only group (Figure 2C). The mice from KA-only group spent less time in the target quadrant than those from control group did, while the mice from Mel+KA group spent comparable time with control group animals in the target quadrant (Figure 2D). Also, the percent distance of Mel+KA group in the target quadrant increased significantly (Figure 2E). Taken together, these results demonstrated that melatonin ameliorates KA-induced memory deficits.

Memory impairment is closely associated with tau hyperphosphorylation (Medeiros et al., 2011), we speculated that tau phosphorylation alleviation might underlie the protective effect of melatonin. Indeed, melatonin treatment significantly lowered the tau phosphorylation at Ser396 and Ser199 in KA-treated mice (Figures $\mathbf{3 A}-\mathbf{C}$ ). We further assayed the distribution of Ser396 hyperphosphorylated tau. KA treatment increased the staining density of p-tau Ser396 in CA1, CA3, and DG regions. The stronger immunoreactivity in the mossy fibers in CA3 sector suggested that the neurons in CA3 region are more sensitive to KA, while the KA-sensitivity of CA1 and DG neurons are relatively mild. Alternatively, these data suggested that the signals of KA may be activated differentially in various regions of the hippocampus. Notably, melatonin decreased the staining density in the CA1, CA3, and DG regions of KA-treated mice hippocampus (Figure 3D). Also, melatonin significantly reduced the phosphorylation level of tau in KA-treated primary hippocampal neurons (Figures 3E,F).

\section{Kainic Acid-Induced Tau Hyperphosphorylation Is Highly Correlated with ER Stress}

Previous reports showed that ER chaperone protein GRP78 level increases in $\mathrm{AD}$ patient brains (Hoozemans et al., 2005). Consistently, the GRP78 level rose in KA-treated mouse hippocampus and reached the highest level at day 7 (Figures 4A,B). Then, we pretreated primary hippocampal neurons with ER stress inhibitor Salubrinal (Sal) for $1 \mathrm{~h}$ and then stimulated with KA for $8 \mathrm{~h}$. With the addition of Sal, the expression levels of GRP78, ATF-6, p-PERK, and IRE-1 significantly decreased (Figures 4C,D), indicating that KA treatment can effectively induce ER stress. 

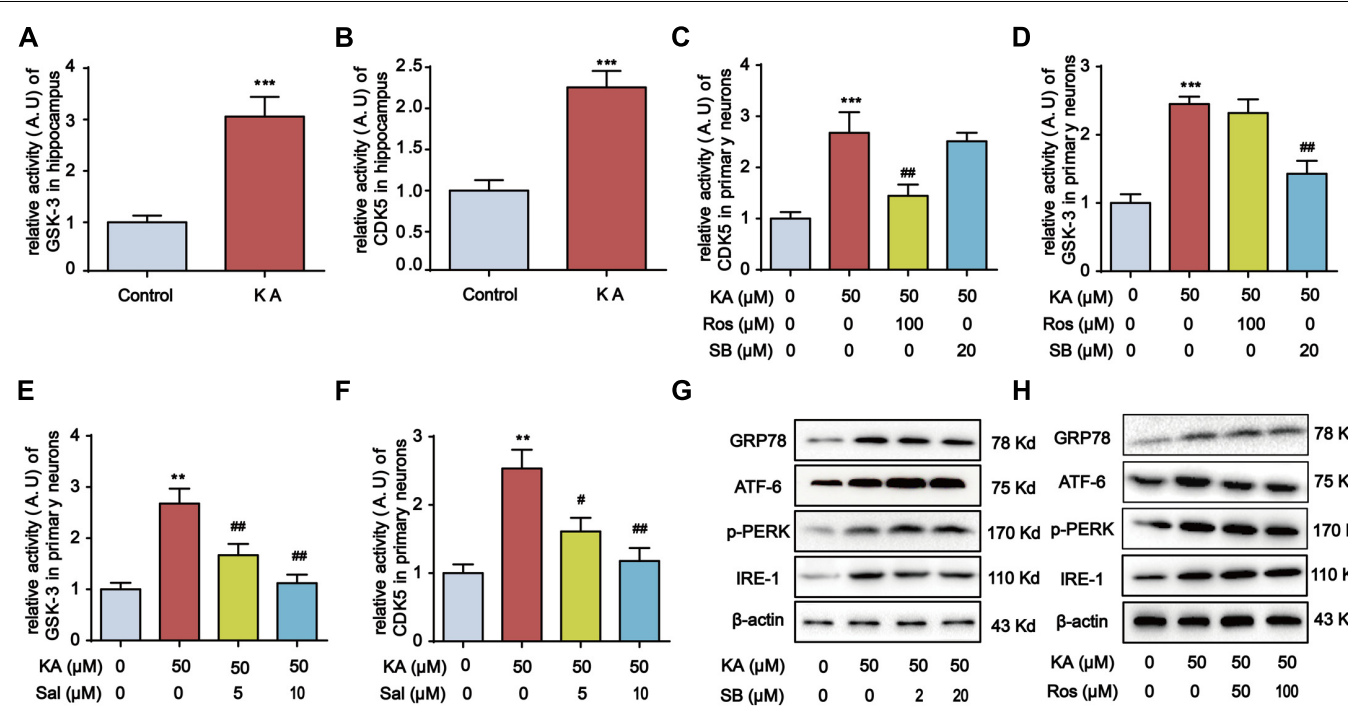

F

G
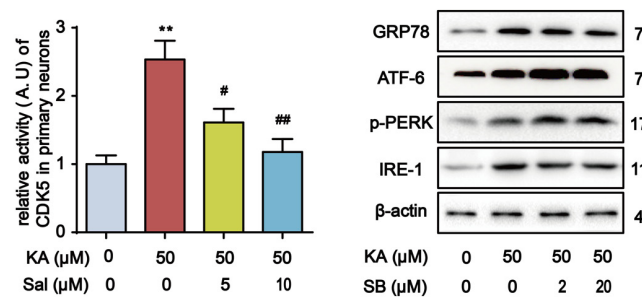

H

1

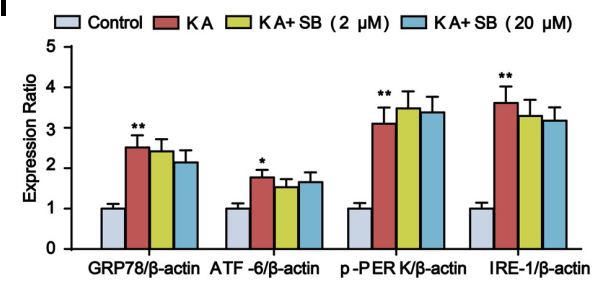

$\mathbf{J}$

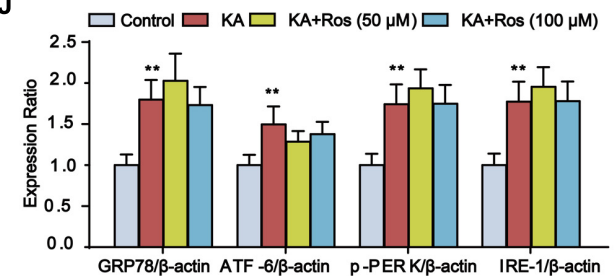

FIGURE 6 | Kainic acid-induced ER stress promotes GSK-3 and CDK5 activities. (A) Relative activity of GSK-3 in KA-treated mouse hippocampus. (B) Relative activity of CDK5 in KA-treated mouse hippocampus. (C) Relative activity of CDK5 in KA, KA+Ros, and/or KA+SB -treated primary hippocampal neurons. The primary hippocampal neurons were pre-treated with or without SB $(20 \mu \mathrm{M})$ or Ros $(100 \mu \mathrm{M})$ for $1 \mathrm{~h}$ and then stimulated with KA (50 $\mu \mathrm{M})$ for $8 \mathrm{~h}$. (D) Relative activity of GSK-3 in KA, KA+Ros, and/or KA+SB -treated primary hippocampal neurons. (E) Relative activity of GSK-3 in KA and/or Sal-treated primary hippocampal neurons. The primary hippocampal neurons were pre-treated with or without Salubrinal (5 or $10 \mu \mathrm{M}$ ) for $1 \mathrm{~h}$ and then stimulated with KA (50 $\mu \mathrm{M})$ for $8 \mathrm{~h}$. (F) Relative activity of CDK5 in KA and/or Sal-treated primary hippocampal neurons. (G,I) Protein levels of GRP78, ATF-6, p-PERK, and IRE-1 in KA and/or SB-treated primary hippocampal neurons. (H,J) Protein levels of GRP78, ATF-6, p-PERK, and IRE-1 in KA and/or Ros-treated primary hippocampal neurons. $\left({ }^{*} P<0.05, * * P<0.01\right.$, ${ }^{* * *} P<0.001$ vs. controls; ${ }^{*} P<0.05$; \#\# $P<0.01$ vs. the KA group; the significant difference from the respective values were determined by one-way analysis of variance test. $N=3$ for western blotting; $n=5$ for assay of activity).

Our recent study showed that ER stress plays an essential role in KA-induced neuronal death (Xue et al., 2017). Thus, we reasoned that ER stress might contribute to KA-induced tau hyperphosphorylation. In parallel with ER stress biomarkers change, tau phosphorylation at Ser199, and Ser396 sites were down-regulated significantly in $\mathrm{KA}+$ Sal neurons $(5$ and $10 \mu \mathrm{M})$ (Figures 4E-G), suggesting that KA-induced ER stress modulates tau phosphorylation.

\section{Kainic Acid-Induced ER Stress Promotes GSK-3 and CDK5 Activities}

GSK-3 $\beta$ and CDK5 are two major protein kinases implicated in AD-like tau hyperphosphorylation, and KA-induced tau hyperphosphorylation is directly associated with the increase in GSK-3 $\beta$ and CDK5 activities (Tripathi et al., 2010). To assay the requirement of GSK-3 and CDK5 in KA-induced tau hyperphosphorylation, we deployed SB216763 (SB, a GSK-3 specific inhibitor) and roscovitine (Ros, a CDK5 specific inhibitor) in KA-treated primary hippocampal neurons, and found that both SB and Ros inhibited tau hyperphosphorylation
(Figure 5). The activity of CDK5 and GSK-3 increased by $\sim 2$ - and $\sim 3$-fold 3 days after KA injection, respectively (Figures 6A,B). Consistently, the activities of CDK5 and GSK-3 were inhibited by roscovitine or SB216763 in KA-treated primary hippocampal neurons, respectively (Figures 6C,D). Notably, in KA and ER stress inhibitor Salubrinal co-treated primary hippocampal neurons, GSK-3 and CDK5 activities were reduced (Figures 6E,F), implying the functional role of ER stress in GSK-3 and CDK5 activities regulation. Furthermore, the upregulated ER stress biomarkers after KA treatment remained intact upon the addition of $\mathrm{SB}$ or Ros (Figures 6G-J), suggesting that ER stress acts upstream of GSK-3 and CDK5 in the process of tau hyperphosphorylation.

\section{Calpain Mediates Kainic Acid-Induced GSK-3 and CDK5 Activation}

Endoplasmic reticulum stress-dependent PERK activation leads to the elevation of the phospho-eIF $2 \alpha$ level, which promotes the preferential synthesis of ATF-4 (Yu et al., 2016). ATF-4 functions boost the expression of calpain, triggering GSK-3 $\beta$ 
A

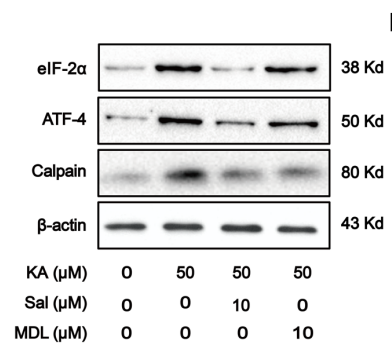

D

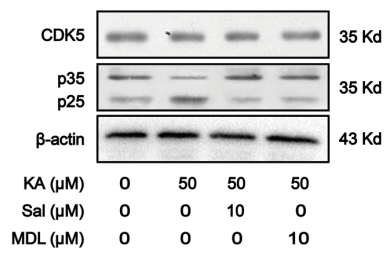

B
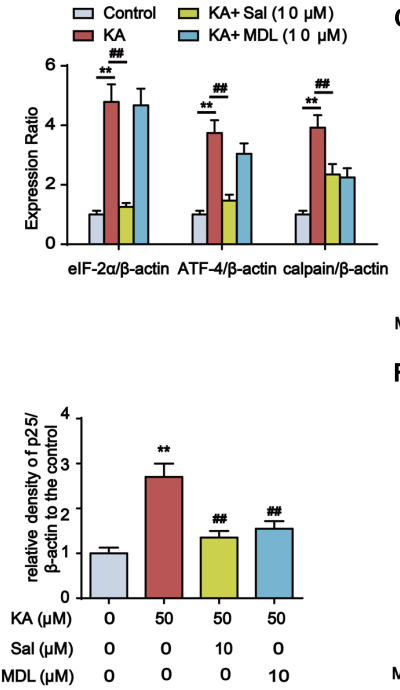

C

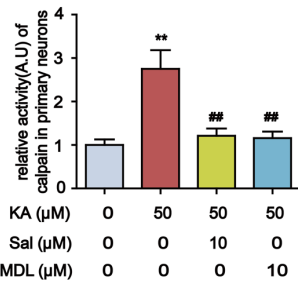

$\mathbf{F}$

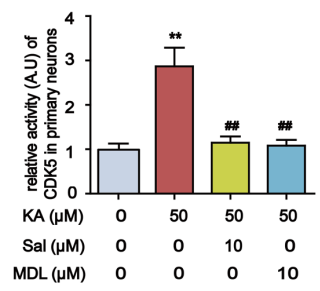

G

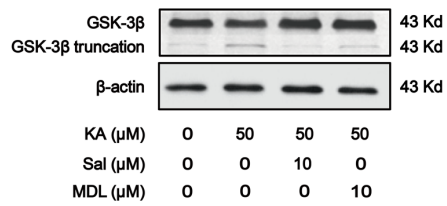

H

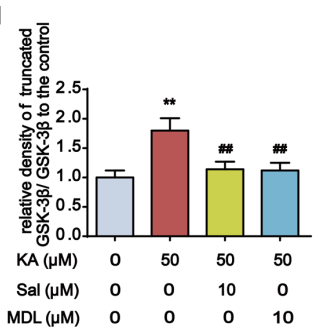

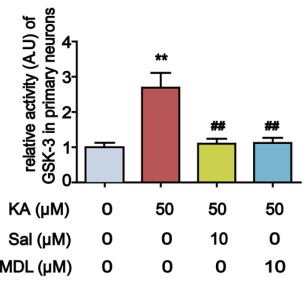

FIGURE 7 | Calpain mediates ER stress-induced GSK-3 $\beta$ and CDK5 activation. (A,B) Protein levels of elF-2 $\alpha$, ATF-4 and calpain in KA, Sal and/or MDL-treated primary hippocampal neurons. The primary hippocampal neurons were pre-treated with or without MDL28170 (MDL, a calpain inhibitor, $10 \mu \mathrm{M}$ ) for $1 \mathrm{~h}$, and then stimulated with KA $(50 \mu \mathrm{M})$ for $8 \mathrm{~h}$. (C) Relative activity of calpain in KA, Sal and/or MDL-treated primary hippocampal neurons. (D,E) Protein levels of CDK5, p35, p25 in KA, Sal and/or MDL-treated primary hippocampal neurons. (F) Relative activity of CDK5 in KA, Sal and/or MDL-treated primary hippocampal neurons. $(\mathbf{G}, \mathbf{H})$ Protein levels of truncated-GSK-3 $\beta$ in KA, Sal and/or MDL-treated primary hippocampal neurons. (I) Relative activity of GSK-3 in KA, Sal and/or MDL-treated primary hippocampal neurons. ${ }^{* *} P<0.01 \mathrm{vs}$. controls; ${ }^{\# \#} P<0.01 \mathrm{vs}$. the KA group; the significant difference from the respective values were determined by one-way analysis of variance test. $n=3$ for western blotting; $N=5$ for assay of activity).

truncation (Han et al., 2014; Lu et al., 2015). Additionally, calpain can catalyze the cleavage of CDK5 neuron-specific activator p35 into more potent p25. Conversion of p35 to p25 would cause prolonged activation and mislocalization of CDK5 (Lee et al., 2000) (Figure 9). Accordingly, we observed that KA treatment prominently increased the expression level of eIF2 $\alpha$, ATF-4, and calpain in primary hippocampal neurons, whereas ER stress inhibitor Salubrinal treatment reduced the expression of them (Figures 7A,B).

To test the requirement of calpain in KA-induced GSK-3 $\beta$ and CDK5 activation, we examined the effect of calpain inhibitor MDL28170 (MDL) on the expression of p25, p35, GSK-3 $\beta$ and CDK5 in primary hippocampal neurons. Compared with KA-only group, Sal+KA or MDL+KA treatment decreased the expression levels of p25 and truncated GSK-3 $\beta$ (Figures 7D,E,G,H). In contrast, KA-induced increase of upstream eIF- $2 \alpha$ or ATF-4 was not affected in calpain-inhibited neurons (MDL+KA treatment) (Figures 7A,B). Further analysis demonstrated that the activities of GSK-3, CDK5, and calpain were downregulated upon the treatment of either Sal or MDL (Figures 7C,F,I). Together our biochemical results suggested that
ER stress-dependent calpain activation underlies KA-induced GSK-3 $\beta$ and CDK5 activation.

\section{Melatonin Inhibits ER Stress, Calpain Activation, and Oxidative Stress}

Thus far, our evidence suggests that KA-induced ER stress triggers GSK-3 $\beta$ and CDK5 activation. To determine whether melatonin inhibits tau hyperphosphorylation via abolishing ER stress, we assayed the expression of proteins involved in ER stress and calpain activation after the treatment of thapsigargin (TG, an ER stress inducer). In both KA- and TG-treated groups, the expression level of p-PERK, ATF-4, eIF-2 $\alpha$, and calpain significantly increased. However, after the addition of melatonin, the expression of p-PERK, ATF-4, eIF-2 $\alpha$, and calpain reduced significantly (Figures $\mathbf{8 A}, \mathbf{B}$ ). Consistently, the increased hippocampal GRP78 level in KA-treated mice could be significantly relieved by melatonin treatment (Figures $\mathbf{8 C}, \mathbf{D}$ ).

It is worth noting that melatonin efficiently hampers the activity of calpain (Figure 8E). We then probed the expression

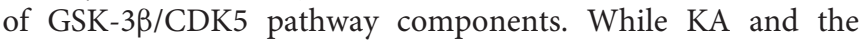
ER stress inducer TG could enhance p25 expression, the 

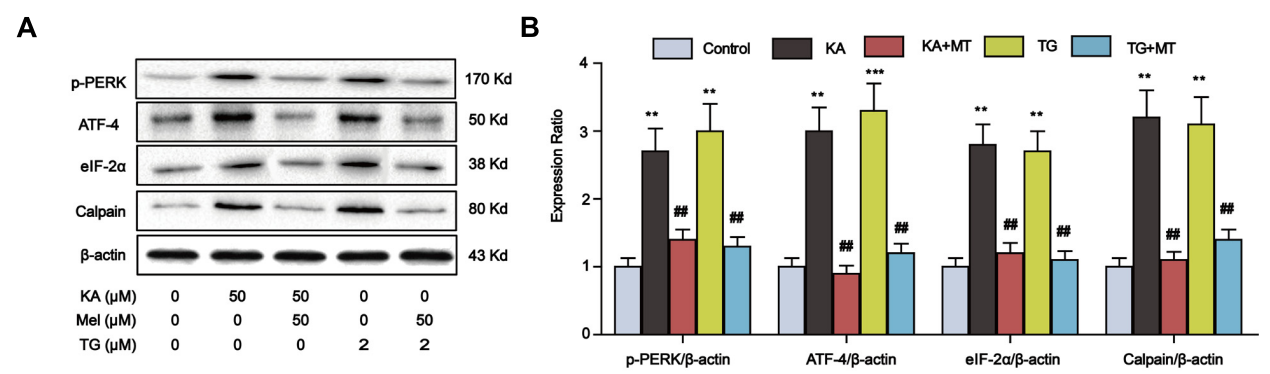

C

D
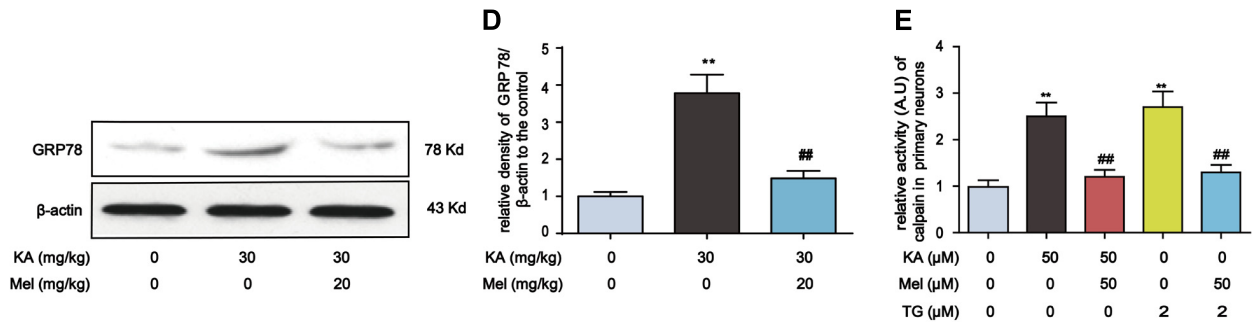

$\mathbf{F}$

G
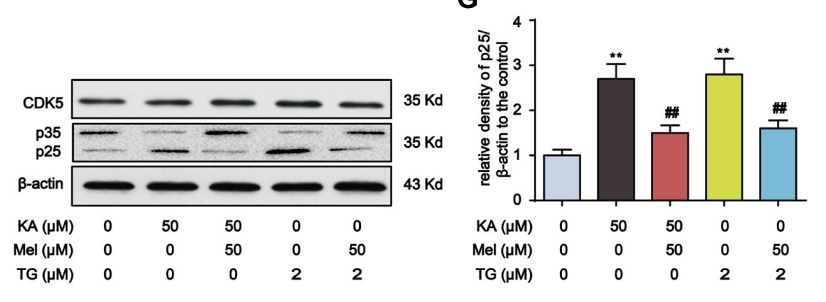

H

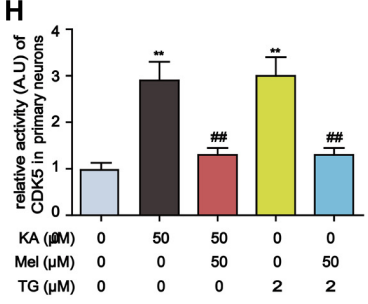

I

$\mathbf{J}$
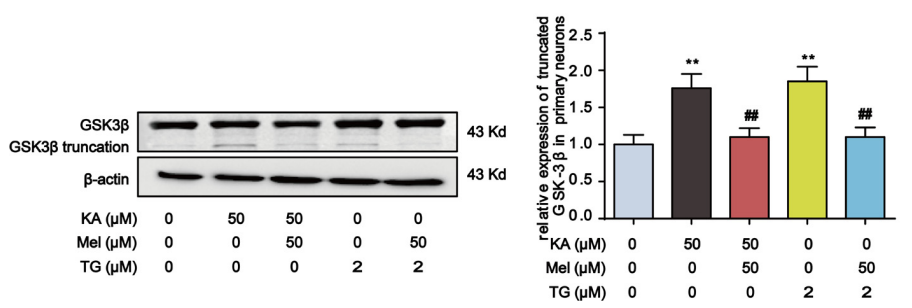

K

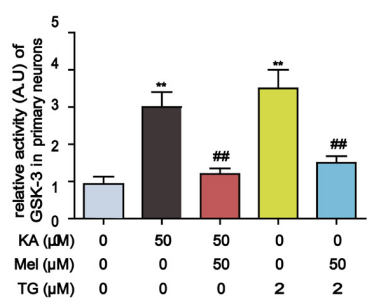

FIGURE 8 | Inhibitory effects of melatonin on ER stress and GSK-3ק/CDK5 activation in primary hippocampal neurons. (A,B) Protein levels of p-PERK, ATF-4, elF-2 $\alpha$ and calpain in KA, TG and/or Mel-treated primary hippocampal neurons. (C,D) Protein levels of GRP78 in KA and/or Mel-treated mouse hippocampus. (E) Relative activity of calpain in KA, TG and/or Mel-treated primary hippocampal neurons. (F,G) Protein levels of CDK5, p35, p25 in KA, TG and/or Mel-treated primary hippocampal neurons. (H) Relative activity of CDK5 in KA, TG and/or Mel-treated primary hippocampal neurons. (I,J) Protein levels of truncated-GSK-3 3 in KA, TG and/or Mel-treated primary hippocampal neurons. (K) Relative activity of GSK-3 in KA, TG and/or Mel-treated primary hippocampal neurons. (** $P<0.01$ vs. controls; ${ }^{\#} P<0.01$ vs. the KA group; the significant difference from the respective values were determined by one-way analysis of variance test. $N=3$ for western blotting, $n=5$ for assay of activity).

level of p25 decreased prominently upon the treatment of melatonin, suggesting the inhibitory effect of melatonin on ER stress-induced CDK5 activation (Figures 8F-H). Furthermore, the increased expression of truncated GSK-3 $\beta$ in KA or TG treated neurons was significantly reduced by melatonin treatment (Figures 8I-K).

Accumulating evidence showed that KA could induce oxidative stress, and melatonin treatment could effectively prevent oxidative damage (Gluck et al., 2000; Pan et al., 2015). Therefore, we tested the level of MDA, an indicator of lipid peroxidation. Also, we analyzed the activity of superoxide dismutase. We noticed that the incubation of primary hippocampal neurons with KA resulted in oxidative stress, which was characterized by a significantly increased level of MDA and a decreased activity of superoxide dismutase. However, when the cells were treated simultaneously with KA and melatonin, the up-regulated level of MDA was significantly decreased, and the activity of superoxide dismutase was considerably boosted (Supplementary Figure S1).

\section{Melatonin Efficiently Eases Intracellular $\mathrm{Ca}^{2+}$ Overload}

As a glutamate analog, $\mathrm{KA}$ is competent to induce $\mathrm{Ca}^{2+}$ overloading by activating glutamate receptors (McGeer and McGeer, 1978). KA-induced extracellular $\mathrm{Ca}^{2+}$ influx leads to cytosolic $\mathrm{Ca}^{2+}$ concentration increase and $\mathrm{ER}$ stress 


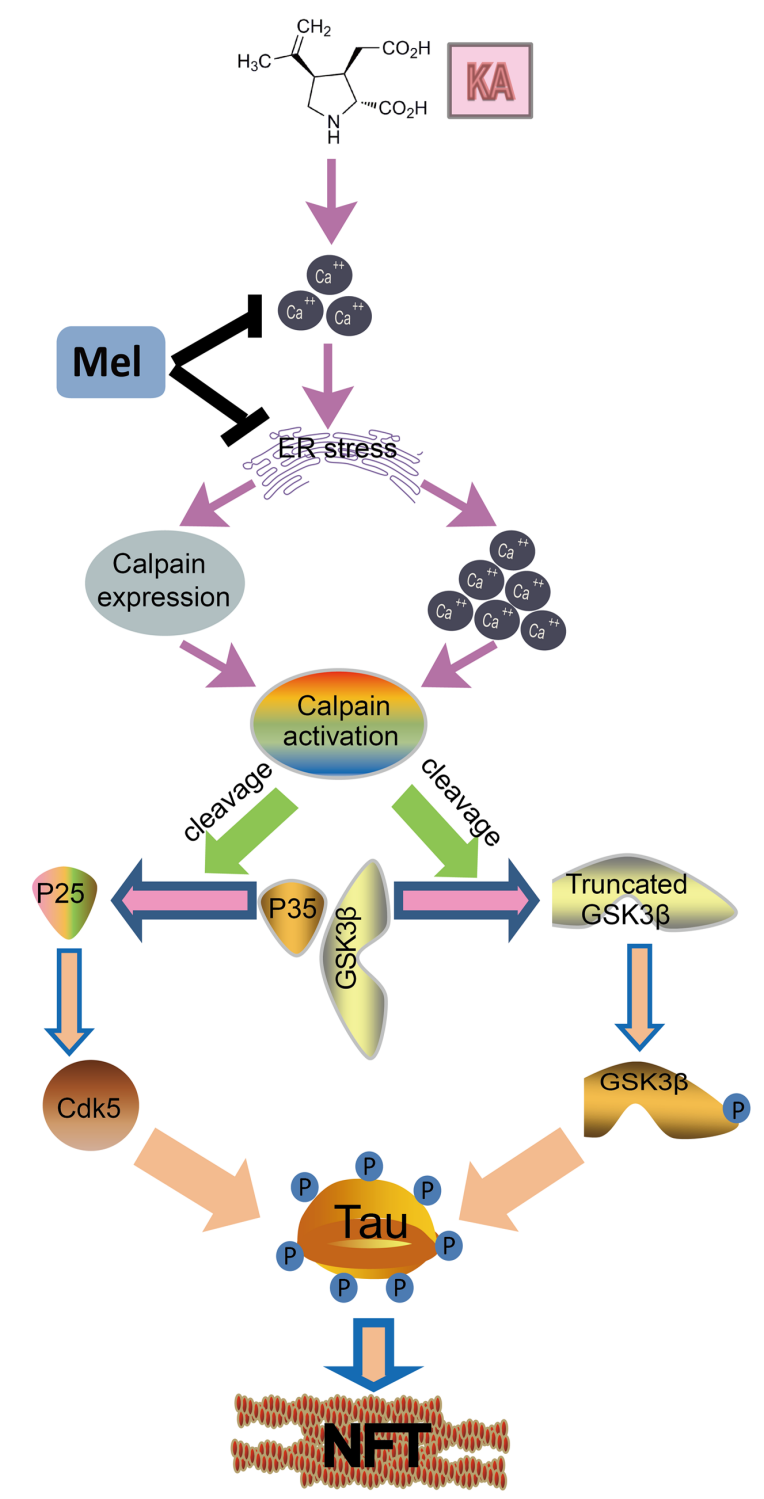

FIGURE 9 | A functional model of melatonin protecting effects against KA-induced neuronal tau hyperphosphorylation and memory deficits. KA treatment triggers ER stress and causes $\mathrm{Ca}^{2+}$ hike, leading to calpain activity upregulation. Upregulated calpain activity eventually results in the cleavage of p35 and GSK-3 $\beta$, and tau hyperphosphorylation. Melatonin inhibits KA-induced GSK-3 $\beta$ and CDK5 activation and tau hyperphosphorylation via alleviating ER stress. Arrows indicate positive regulation, and blocks indicate negative regulation.

(Schinder et al., 1996; Nicholls, 2004; Kim et al., 2016). Furthermore, ER dysfunction promotes $\mathrm{Ca}^{2+}$ release from ER and aggravate cytosolic $\mathrm{Ca}^{2+}$ hike (Bahar et al., 2016). To investigate the effect of melatonin on intracellular $\mathrm{Ca}^{2+}$ homeostasis after KA treatment, we monitored intracellular $\mathrm{Ca}^{2+}$ concentrations with Fura-2 AM. Supplementary Figure S3A shows the concentrations profile of $\mathrm{Ca}^{2+}$. KA treatment led to significant increases in cytosolic $\mathrm{Ca}^{2+}$ levels in both KA group and KA+Mel group concerning both amplitude and duration.
However, the treatment of melatonin remarkably damper peak amplitude (5.13 \pm 0.44 vs. $3.38 \pm 0.25, n=5, p<0.05)$. In addition, we calculated the AUC for $\mathrm{Ca}^{2+}$ concentration over $8 \mathrm{~h}$, and the results showed that melatonin significantly reduced the AUC by approximately $40 \%$ compared to the KA group (Supplementary Figure S3B). Therefore, melatonin could mitigate the KA-induced calpain activation by alleviating $\mathrm{Ca}^{2+}$ overload.

Due to the contribution of both internal and external sources of $\mathrm{Ca}^{2+}$, it is intriguing to determine whether ER stress-induced ER $\mathrm{Ca}^{2+}$ release significantly contributes to calpain activation. To this end, we used three intracellular $\mathrm{Ca}^{2+}$ modulators to explore the roles of $\left[\mathrm{Ca}^{2+}\right]_{\mathrm{c}}\left(\right.$ cytosolic $\left.\mathrm{Ca}^{2+}\right)$ and $\left[\mathrm{Ca}^{2+}\right]_{\mathrm{ER}}(\mathrm{ER}$ $\mathrm{Ca}^{2+}$ pool) in KA-treated primary hippocampal neurons. 2-APB is a specific inhibitor of inositol 1, 4, 5-trisphosphate receptor (IP3R) that promotes $\mathrm{Ca}^{2+}$ release from ER (Yoon et al., 2014). TG can elevate cytosolic $\mathrm{Ca}^{2+}$ concentration by inhibiting the ER-Ca ${ }^{2+}$-ATPase (Abdoul-Azize et al., 2017), while BAPTA-AM is an intracellular $\mathrm{Ca}^{2+}$ chelator (Liu et al., 2016). Primary hippocampal neurons were pretreated with $10 \mu \mathrm{M}$ BAPTA-AM for $30 \mathrm{~min}$, followed by the incubation with $50 \mu \mathrm{M} \mathrm{KA}$ for additional $8 \mathrm{~h}$. Similarly, primary hippocampal neurons were co-incubated with $10 \mu \mathrm{M}$ 2-APB or $1 \mu \mathrm{M}$ TG and KA for $8 \mathrm{~h}$. We noticed that treatment with 2-APB significantly inhibited KA-induced $\left[\mathrm{Ca}^{2+}\right]_{\mathrm{ER}}$ depletion (Supplementary Figure S3D) and suppressed $\left[\mathrm{Ca}^{2+}\right]_{c}$ elevation (Supplementary Figure S3C). Instead, treatment with BAPTA-AM suppressed KA-induced $\left[\mathrm{Ca}^{2+}\right]_{\mathrm{c}}$ elevation while does not affect $\left[\mathrm{Ca}^{2+}\right]_{\mathrm{ER}}$ release (Supplementary Figures S3C,D). Moreover, treatment with TG further aggravated KA-mediated cellular $\mathrm{Ca}^{2+}$ overloading (Supplementary Figure S3C). These results suggested that KA can efficiently induce ER stress and $\mathrm{Ca}^{2+}$ release from the ER. Consistently, both the protein level and the activity of calpain were up-regulated synchronously with a KA-mediated cellular $\mathrm{Ca}^{2+}$ increase (Figures 8A,E). Therefore, although we lack direct evidence showing $\left[\mathrm{Ca}^{2+}\right]_{\mathrm{ER}}$ release by ER stress leads to an upregulation of calpain activity, we speculate that KA-induced ER stress plays an indispensable role in calpain activation via promoting $\left[\mathrm{Ca}^{2+}\right]_{\mathrm{c}}$.

\section{DISCUSSION}

The abnormally hyperphosphorylated tau is the major component of intracellular NFTs, which is positively correlated with the decline of memory and cognition in $\mathrm{AD}$ patients (Shipton et al., 2011). Multiple factors have been reported to play roles in the etiology of $\mathrm{AD}$ regarding initiation and progression. The excitotoxicity might occur in the early stage of the $\mathrm{AD}$ and contribute to the neurodegenerative process (Pallo et al., 2016). In the current study, we demonstrated that KA treatment induced ER stress and activated GSK$3 \beta$ and CDK5. Moreover, we showed that KA-induced ER stress significantly activated calpain. In addition, GSK-3 $\beta$ or CDK5 inhibition failed to reduce ER stress, suggesting that ER stress occurs upstream of GSK-3 $\beta$ or CDK5. Importantly, we found that melatonin can appreciably ease KA-induced 
tau hyperphosphorylation and cognition disabilities via manipulating ER stress-mediated signaling pathway. In the meantime, we also noticed that the level of tau phosphorylation starts to decrease at day 7 after KA administration (Figure 1A). Nevertheless, ER chaperone protein GRP78 level continues to increase after day 7 (Figure 4A). This discrepancy could be attributed to the subsequent activation of phosphatase $2 \mathrm{~A}$ (PP2A), leading to enhanced tau dephosphorylation. Indeed, previous studies showed that there is a dynamic fluctuation in the activity of PP2A in the KA-treated mouse brain (Liang et al., 2009).

Endoplasmic reticulum stress has been shown to contribute to various disorders in the central nervous system (Omura et al., 2013). In AD brains, p-PERK immunoreactivity was most abundant in the neurons with diffusive localization of phosphorylated tau (Hoozemans et al., 2009). Also, constant illumination resulted in ER damage, together with the decreased melatonin secretion, upregulated tau hyperphosphorylation and memory impairment (Ling et al., 2009). These studies implied the participation of ER stress in the pathophysiological process of the AD. Accordingly, our results demonstrated that the inhibition of ER stress efficiently downregulated tau hyperphosphorylation and the activation of GSK-3 $\beta$ and CDK5. Calpain-mediated CDK5/p35 cleavage into CDK5/p25 is necessary for tau phosphorylation (Chen et al., 2008). GSK-3 $\beta$ C-terminal truncation is also correlated with the over-activation of calpain in AD brains (Jin et al., 2015). ER stress-dependent activation of PERK leads to significant elevation of phospho-eIF $2 \alpha$, which attenuates general translation and promotes the preferential synthesis of ATF-4. ATF-4 then causes increased expression of calpain, hence triggers the proteasomal degradation of p35 and GSK-3 $\beta$ truncation (de la Cadena et al., 2014).

The calpain activity can be attributable to two main isoforms (calpain I and calpain II) which differed primarily in their calcium requirements. In the present study, we demonstrated that KA could simotaneouly induce cytosolic $\mathrm{Ca}^{2+}$ elevation and ER $\mathrm{Ca}^{2+}$ release. Moreover, melatonin is capable of decreasing cytosolic $\mathrm{Ca}^{2+}$ and $\mathrm{ER} \mathrm{Ca}^{2+}$ release in KA-treated primary hippocampal neurons. Thus, we speculate that KA-induced ER stress plays an indispensable role in calpain activation via promoting cytosolic $\mathrm{Ca}^{2+}$ level.

It was reported that calpain I is correlated with the excitatory amino acid-induced hippocampal damage and acts as an intracellular mediator within the process (Siman et al., 1989). In the present study, we observed that p-PERKelF2 $\alpha$-ATF-4 pathway mediates KA-induced upregulation of calpain, and the elevated calpain activity contributes to the activation of CDK5/GSK-3 $\beta$ and tau hyperphosphorylation, fortifying the mechanistic role of calpain connecting tau hyperphosphorylation with KA-induced ER stress. We also examined the role of calpain I in KA-induced tau hyperphosphorylation in N2a cells. As shown in Supplementary Figure S2, transfection of calpain I siRNA led to a significant abatement in KA-induced tau hyperphosphorylation, indicating that calpain I activation is involved in the KA-induced ER stress and tau hyperphosphorylation.
It has been speculated that tau hyperphosphorylation is a response to apoptosis. However, previous studies also showed that hippocampal neurons could survive with NFTs for over 20 years (Morsch et al., 1999). In P301S model mice, although their neurons lack cell apoptosis, there is significant tau hyperphosphorylation (Allen et al., 2002). Therefore, the correlation between tau hyperphosphorylation and apoptosis remains controversial. Unexpectedly, the pro-apoptotic factorsinduced tau hyperphosphorylation was found to be accompanied by the reduced cell apoptosis ( $\mathrm{Li}$ et al., 2007). Thus, it has been proposed that tau hyperphosphorylation may play a role in leading the neurons to abort from an acute apoptosis and triggering a chronic degenerative cell death simultaneously (Wang et al., 2014). Consistently, although prolonged ER stress could cause apoptosis, tau phosphorylation attenuated ER stress-induced apoptosis by upregulating UPR (Liu et al., 2012). Specifically, in mouse hippocampus, the activity of GSK3 $\beta / C D K 5$ increased 1-3 days after KA treatment. After 7 days, a reduction in GSK3 $\beta /$ CDK5 activation was observed, and caspase- 3 activity increased. Combined with our previous findings (Xue et al., 2017), it is possible that KA-induced tau phosphorylation occurs upstream of apoptosis.

Alzheimer's disease is the most common cause of dementia, and there is no specific and efficient cure for this disorder. Accumulating evidence suggests that melatonin plays a critical role in the development of $\mathrm{AD}$ and serves as a potential candidate for arresting AD-like pathological processes (Rudnitskaya et al., 2015). Melatonin can ease KA-induced tau hyperphosphorylation by inhibiting GSK-3 $\beta$ and CDK5 (Peng et al., 2013). Also, melatonin was reported to deactivate ER stress-mediated PERK-eIF2 $\alpha$-ATF-4 (Yu et al., 2016). However, it is not clear whether melatonin exerts its inhibitory effects on KA-induced tau phosphorylation and memory deficits via ER stress revising in vivo. Our studies showed that melatonin has beneficial effects on tau phosphorylation and memory deficits. Melatonin treatment greatly decreased the level of intracellular $\mathrm{Ca}^{2+}$ in KA-treated neurons. Moreover, melatonin treatment significantly reduced calpain expression/activation and ER stress in primary hippocampal neurons. Based on these results, we speculate that the cytoprotective role of melatonin could be attributed to the inhibition of ER stress and p-PERK/eIF- $\alpha / A T F-4 /$ calpain pathway. In addition, reactive oxygen has been extensively reported to induce the deterioration of ER function and ER stress. Notably, prolonged ER stress also provoked oxidative stress, causing a toxic accumulation of ROS within the cell (Cao and Kaufman, 2014; Chaudhari et al., 2014). Thus, ER stress and oxidative stress are likely to be mutually reinforcing. Our data suggested that the antioxidative effect of melatonin could contribute to the ease of tau hyperphosphorylation. Melatonin partially reversed the KA-induced MDA elevation and the KA-suppressed superoxide dismutase activity. However, vitamin E, a recognized antioxidant, failed to affect the activities of CDK5/GSK3 $\beta$ and the hyperphosphorylation level of tau (data not shown), indicating that the antioxidative effect of melatonin is not sufficient to address its protective functionality. 


\section{AUTHOR CONTRIBUTIONS}

CS and JZ carried out the cell culture and biochemical measurements, and drafted the manuscript. ZL and QC carried out the animal treatment and experiments. WH and LX carried out the GSK-3 activity assay. YW carried out the calpain activity assay. AS and JC conceived the study and participated in the coordination and preparation of the manuscript. All authors read and approved the final draft.

\section{FUNDING}

This work was supported by the National Natural Science Foundation of China $(81371416,31670778,31171027)$, the Fundamental Research Funds for the Central Universities (HUST: 2016YXMS191, 2014YGYL005) to JC, the National Natural Science Foundation of China (31771570, 31571466, 81371418), the Program for HUST Interdisciplinary Innovation

\section{REFERENCES}

Abdoul-Azize, S., Dubus, I., and Vannier, J. P. (2017). Improvement of dexamethasone sensitivity by chelation of intracellular $\mathrm{Ca}^{2+}$ in pediatric acute lymphoblastic leukemia cells through the prosurvival kinase ERK1/2 deactivation. Oncotarget 8, 27339-27352. doi: 10.18632/oncotarget.16039

Acuna-Castroviejo, D., Escames, G., Venegas, C., Diaz-Casado, M. E., LimaCabello, E., Lopez, L. C., et al. (2014). Extrapineal melatonin: sources, regulation, and potential functions. Cell. Mol. Life Sci. 71, 2997-3025. doi: 10.1007/s00018-014-1579-2

Allen, B., Ingram, E., Takao, M., Smith, M. J., Jakes, R., Virdee, K., et al. (2002). Abundant tau filaments and nonapoptotic neurodegeneration in transgenic mice expressing human P301S tau protein. J. Neurosci. 22, 9340-9351.

Bahar, E., Kim, H., and Yoon, H. (2016). ER stress-mediated signaling: action potential and $\mathrm{Ca}^{2+}$ as key players. Int. J. Mol. Sci. 17:E1558. doi: 10.3390/ ijms 17091558

Brusco, L. I., Marquez, M., and Cardinali, D. P. (2000). Melatonin treatment stabilizes chronobiologic and cognitive symptoms in Alzheimer's disease. Neuro Endocrinol. Lett. 21, 39-42.

Cao, S. S., and Kaufman, R. J. (2014). Endoplasmic reticulum stress and oxidative stress in cell fate decision and human disease. Antioxid. Redox. Signal. 21, 396-413. doi: 10.1089/ars.2014.5851

Chaudhari, N., Talwar, P., Parimisetty, A., Lefebvre D'hellencourt, C., and Ravanan, P. (2014). A molecular web: endoplasmic reticulum stress, inflammation, and oxidative stress. Front. Cell. Neurosci. 8:213. doi: 10.3389/ fncel.2014.00213

Chen, X., Huang, T., Zhang, J., Song, J., Chen, L., and Zhu, Y. (2008). Involvement of calpain and p25 of CDK5 pathway in ginsenoside Rbl's attenuation of betaamyloid peptide25-35-induced tau hyperphosphorylation in cortical neurons. Brain Res. 1200, 99-106. doi: 10.1016/j.brainres.2007.12.029

Choi, D. W., and Rothman, S. M. (1990). The role of glutamate neurotoxicity in hypoxic-ischemic neuronal death. Annu. Rev. Neurosci. 13, 171-182. doi: 10.1146/annurev.ne.13.030190.001131

Crespo-Biel, N., Camins, A., Canudas, A. M., and Pallas, M. (2010). Kainateinduced toxicity in the hippocampus: potential role of lithium. Bipolar Disord. 12, 425-436. doi: 10.1111/j.1399-5618.2010.00825.x

de la Cadena, S. G., Hernandez-Fonseca, K., Camacho-Arroyo, I., and Massieu, L. (2014). Glucose deprivation induces reticulum stress by the PERK pathway and caspase-7- and calpain-mediated caspase-12 activation. Apoptosis 19, 414-427. doi: 10.1007/s10495-013-0930-7

Gluck, M. R., Jayatilleke, E., Shaw, S., Rowan, A. J., and Haroutunian, V. (2000). CNS oxidative stress associated with the kainic acid rodent model of
Team and the Fundamental Research Funds for the Central University (2016JCTD108), the Specialized Research Fund for the Doctoral Program of Higher Education (20130142110071), the Program for New Century Excellent Talents in University (NCET-13-0234), and the Junior Thousand Talents Program of China to AS.

\section{ACKNOWLEDGMENTS}

We thank Dong Li, Zhenrong Yang, and Xin Fu for the technical assistance.

\section{SUPPLEMENTARY MATERIAL}

The Supplementary Material for this article can be found online at: https://www.frontiersin.org/articles/10.3389/fnmol. 2018.00005/full\#supplementary-material

experimental epilepsy. Epilepsy Res. 39, 63-71. doi: 10.1016/S0920-1211(99) 00111-4

Grundke-Iqbal, I., Iqbal, K., Quinlan, M., Tung, Y. C., Zaidi, M. S., and Wisniewski, H. M. (1986). Microtubule-associated protein tau. A component of Alzheimer paired helical filaments. J. Biol. Chem. 261, 6084-6089.

Hadj Ayed Tka, K., Mahfoudh Boussaid, A., Zaouali, M. A., Kammoun, R., Bejaoui, M., Ghoul Mazgar, S., et al. (2015). Melatonin modulates endoplasmic reticulum stress and Akt/GSK3-beta signaling pathway in a rat model of renal warm ischemia reperfusion. Anal. Cell. Pathol. 2015:635172. doi: 10.1155/2015/ 635172

Han, G., Casson, R. J., Chidlow, G., and Wood, J. P. (2014). The mitochondrial complex I inhibitor rotenone induces endoplasmic reticulum stress and activation of GSK-3beta in cultured rat retinal cells. Invest. Ophthalmol. Vis. Sci. 55, 5616-5628. doi: 10.1167/iovs.14-14371

Hernandez, F., and Avila, J. (2008). Tau aggregates and tau pathology. J. Alzheimers Dis. 14, 449-452. doi: 10.3233/JAD-2008-14414

Hoozemans, J. J. M., Van Haastert, E. S., Nijholt, D. A. T., Rozemuller, A. J. M., Eikelenboom, P., and Scheper, W. (2009). The unfolded protein response is activated in pretangle neurons in Alzheimer's disease hippocampus. Am. J. Pathol. 174, 1241-1251. doi: 10.2353/ajpath.2009.080814

Hoozemans, J. J. M., Veerhuis, R., Van Haastert, E. S., Rozemuller, J. M., Baas, F., Eikelenboom, P., et al. (2005). The unfolded protein response is activated in Alzheimer's disease. Acta Neuropathol. 110, 165-172. doi: 10.1007/s00401-0051038-0

Isaev, N. K., Stelmashook, E. V., Dirnagl, U., Andreeva, N. A., Manuhova, L., Vorobjev, V. S., et al. (2002). Neuroprotective effects of the antifungal drug clotrimazole. Neuroscience 113, 47-53. doi: 10.1016/S0306-4522(02)00164-1

Jain, A., Sharma, D., Suhalka, P., Sukhwal, P., and Bhatnagar, M. (2013). Changes in the density of nitrergic neurons in the hippocampus of rats following kainic acid and melatonin administration. Physiol. Res. 62, 197-203.

Jin, N., Yin, X., Yu, D., Cao, M., Gong, C. X., Iqbal, K., et al. (2015). Truncation and activation of GSK-3beta by calpain I: a molecular mechanism links to tau hyperphosphorylation in Alzheimer's disease. Sci. Rep. 5:8187. doi: 10.1038/ srep08187

Kim, H., Lee, J. Y., Park, K. J., Kim, W. H., and Roh, G. S. (2016). A mitochondrial division inhibitor, Mdivi-1, inhibits mitochondrial fragmentation and attenuates kainic acid-induced hippocampal cell death. BMC Neurosci. 17:33. doi: 10.1186/s12868-016-0270-y

Kim, J. S., Heo, R. W., Kim, H., Yi, C. O., Shin, H. J., Han, J. W., et al. (2014). Salubrinal, ER stress inhibitor, attenuates kainic acid-induced hippocampal cell death. J. Neural. Transm. 121, 1233-1243. doi: 10.1007/s00702-014-1208-0

Kobe, H., Nakai, A., Koshino, T., and Araki, T. (2002). Effect of regular maternal exercise on lipid peroxidation levels and antioxidant enzymatic activities 
before and after delivery. J. Nippon Med. Sch. 69, 542-548. doi: 10.1272/jnms. 69.542

Lee, M. S., Kwon, Y. T., Li, M., Peng, J., Friedlander, R. M., and Tsai, L. H. (2000). Neurotoxicity induces cleavage of p 35 to p 25 by calpain. Nature $405,360-364$. doi: $10.1038 / 35012636$

Li, H. L., Wang, H. H., Liu, S. J., Deng, Y. Q., Zhang, Y. J., Tian, Q., et al. (2007). Phosphorylation of tau antagonizes apoptosis by stabilizing beta-catenin, a mechanism involved in Alzheimer's neurodegeneration. Proc. Natl. Acad. Sci. U.S.A. 104, 3591-3596. doi: 10.1073/pnas.0609303104

Liang, Z., Liu, F., Iqbal, K., Grundke-Iqbal, I., and Gong, C. X. (2009). Dysregulation of tau phosphorylation in mouse brain during excitotoxic damage. J. Alzheimers Dis. 17, 531-539. doi: 10.3233/JAD-2009-1069

Ling, Z. Q., Tian, Q., Wang, L., Fu, Z. Q., Wang, X. C., Wang, Q., et al. (2009). Constant illumination induces Alzheimer-like damages with endoplasmic reticulum involvement and the protection of melatonin. J. Alzheimers Dis. 16, 287-300. doi: 10.3233/JAD-2009-0949

Liu, C., Ye, Y., Zhou, Q., Zhang, R., Zhang, H., Liu, W., et al. (2016). Crosstalk between $\mathrm{Ca}^{2+}$ signaling and mitochondrial $\mathrm{H}_{2} \mathrm{O}_{2}$ is required for rotenone inhibition of mTOR signaling pathway leading to neuronal apoptosis. Oncotarget 7, 7534-7549. doi: 10.18632/oncotarget.7183

Liu, R. Y., Zhou, J. N., Van Heerikhuize, J., Hofman, M. A., and Swaab, D. F. (1999). Decreased melatonin levels in postmortem cerebrospinal fluid in relation to aging, Alzheimer's disease, and apolipoprotein E-epsilon4/4 genotype. J. Clin. Endocrinol. Metab. 84, 323-327.

Liu, X. A., Song, J., Jiang, Q., Wang, Q., Tian, Q., and Wang, J. Z. (2012). Expression of the hyperphosphorylated tau attenuates ER stress-induced apoptosis with upregulation of unfolded protein response. Apoptosis 17, 1039-1049. doi: 10.1007/s10495-012-0744-z

Lu, T. H., Su, C. C., Tang, F. C., Chen, C. H., Yen, C. C., Fang, K. M., et al. (2015). Chloroacetic acid triggers apoptosis in neuronal cells via a reactive oxygen species-induced endoplasmic reticulum stress signaling pathway. Chem. Biol. Interact. 225, 1-12. doi: 10.1016/j.cbi.2014.10.022

Marklund, S., and Marklund, G. (1974). Involvement of the superoxide anion radical in the autoxidation of pyrogallol and a convenient assay for superoxide dismutase. Eur. J. Biochem. 47, 469-474. doi: 10.1111/j.1432-1033.1974. tb03714.x

McGeer, E. G., and McGeer, P. L. (1978). Some factors influencing the neurotoxicity of intrastriatal injections of kainic acid. Neurochem. Res. 3, 501-517. doi: 10.1007/BF00966331

Medeiros, R., Baglietto-Vargas, D., and Laferla, F. M. (2011). The role of tau in Alzheimer's disease and related disorders. CNS Neurosci. Ther. 17, 514-524. doi: 10.1111/j.1755-5949.2010.00177.x

Milatovic, D., Zivin, M., Gupta, R. C., and Dettbarn, W. D. (2001). Alterations in cytochrome coxidase activity and energy metabolites in response to kainic acidinduced status epilepticus. Brain Res. 912, 67-78. doi: 10.1016/S0006-8993(01) 02657-9

Morris, M., Maeda, S., Vossel, K., and Mucke, L. (2011). The many faces of tau. Neuron 70, 410-426. doi: 10.1016/j.neuron.2011.04.009

Morsch, R., Simon, W., and Coleman, P. D. (1999). Neurons may live for decades with neurofibrillary tangles. J. Neuropathol. Exp. Neurol. 58, 188-197. doi: 10.1097/00005072-199902000-00008

Nicholls, D. G. (2004). Mitochondrial dysfunction and glutamate excitotoxicity studied in primary neuronal cultures. Curr. Mol. Med. 4, 149-177. doi: 10.2174/ 1566524043479239

Omura, T., Kaneko, M., Okuma, Y., Matsubara, K., and Nomura, Y. (2013). Endoplasmic reticulum stress and Parkinson's disease: the role of HRD1 in averting apoptosis in neurodegenerative disease. Oxid. Med. Cell. Longev. 2013:239854. doi: 10.1155/2013/239854

Pallo, S. P., Dimaio, J., Cook, A., Nilsson, B., and Johnson, G. V. (2016). Mechanisms of tau and Abeta-induced excitotoxicity. Brain Res. 1634, 119-131. doi: 10.1016/j.brainres.2015.12.048

Pan, X., Zhu, L., Lu, H., Wang, D., Lu, Q., and Yan, H. (2015). Melatonin attenuates oxidative damage induced by acrylamide in vitro and in vivo. Oxid. Med. Cell. Longev. 2015:703709. doi: 10.1155/2015/703709

Peng, C. X., Hu, J., Liu, D., Hong, X. P., Wu, Y. Y., Zhu, L. Q., et al. (2013). Disease-modified glycogen synthase kinase-3beta intervention by melatonin arrests the pathology and memory deficits in an Alzheimer's animal model. Neurobiol. Aging 34, 1555-1563. doi: 10.1016/j.neurobiolaging.2012. 12.010
Pigino, G., Paglini, G., Ulloa, L., Avila, J., and Caceres, A. (1997). Analysis of the expression, distribution and function of cyclin dependent kinase 5 (cdk5) in developing cerebellar macroneurons. J. Cell Sci. 110( Pt 2), 257-270.

Rudnitskaya, E. A., Muraleva, N. A., Maksimova, K. Y., Kiseleva, E., Kolosova, N. G., and Stefanova, N. A. (2015). Melatonin attenuates memory impairment, amyloid-beta accumulation, and neurodegeneration in a rat model of sporadic Alzheimer's disease. J. Alzheimers Dis. 47, 103-116. doi: 10.3233/JAD- 150161

Salminen, A., Kauppinen, A., Suuronen, T., Kaarniranta, K., and Ojala, J. (2009). ER stress in Alzheimer's disease: a novel neuronal trigger for inflammation and Alzheimer's pathology. J. Neuroinflammation 6:41. doi: 10.1186/17422094-6-41

Schinder, A. F., Olson, E. C., Spitzer, N. C., and Montal, M. (1996). Mitochondrial dysfunction is a primary event in glutamate neurotoxicity. J. Neurosci. 16, 6125-6133.

Sharma, S., Sarkar, J., Haldar, C., and Sinha, S. (2014). Melatonin reverses Fas, E2F1 and endoplasmic reticulum stress mediated apoptosis and dysregulation of autophagy induced by the herbicide atrazine in murine splenocytes. PLOS ONE 9:e108602. doi: 10.1371/journal.pone.0108602

Shipton, O. A., Leitz, J. R., Dworzak, J., Acton, C. E. J., Tunbridge, E. M., Denk, F., et al. (2011). Tau protein is required for amyloid $\beta$-induced impairment of hippocampal long-term potentiation. J. Neurosci. 31, 1688-1692. doi: 10.1523/ JNEUROSCI.2610-10.2011

Siman, R., Noszek, J. C., and Kegerise, C. (1989). Calpain I activation is specifically related to excitatory amino acid induction of hippocampal damage. J. Neurosci. 9, 1579-1590.

Tripathi, P. P., Santorufo, G., Brilli, E., Borrelli, E., and Bozzi, Y. (2010). Kainic acid-induced seizures activate GSK-3beta in the hippocampus of D2R-/- mice. Neuroreport 21, 846-850. doi: 10.1097/WNR.0b013e32833d5891

Wang, H., Wang, Z. K., Jiao, P., Zhou, X. P., Yang, D. B., Wang, Z. Y., et al. (2015). Redistribution of subcellular calcium and its effect on apoptosis in primary cultures of rat proximal tubular cells exposed to lead. Toxicology 333, 137-146. doi: 10.1016/j.tox.2015.04.015

Wang, J. Z., Wang, Z. H., and Tian, Q. (2014). Tau hyperphosphorylation induces apoptotic escape and triggers neurodegeneration in Alzheimer's disease. Neurosci. Bull. 30, 359-366. doi: 10.1007/s12264-013-1415-y

$\mathrm{Wu}$, Y. H., and Swaab, D. F. (2005). The human pineal gland and melatonin in aging and Alzheimer's disease. J. Pineal Res. 38, 145-152. doi: 10.1111/j.1600079X.2004.00196.X

Xue, F., Shi, C., Chen, Q., Hang, W., Xia, L., Wu, Y., et al. (2017). Melatonin mediates protective effects against kainic acid-induced neuronal death through safeguarding ER stress and mitochondrial disturbance. Front. Mol. Neurosci. 10:49. doi: 10.3389/fnmol.2017.00049

Yoon, M. J., Lee, A. R., Jeong, S. A., Kim, Y. S., Kim, J. Y., Kwon, Y. J., et al. (2014). Release of $\mathrm{Ca}^{2+}$ from the endoplasmic reticulum and its subsequent influx into mitochondria trigger celastrol-induced paraptosis in cancer cells. Oncotarget 5 , 6816-6831. doi: 10.18632/oncotarget.2256

Yu, L., Li, B., Zhang, M., Jin, Z., Duan, W., Zhao, G., et al. (2016). Melatonin reduces PERK-eIF2alpha-ATF4-mediated endoplasmic reticulum stress during myocardial ischemia-reperfusion injury: role of RISK and SAFE pathways interaction. Apoptosis 21, 809-824. doi: 10.1007/s10495-016-1246-1

Zhang, X. M., and Zhu, J. (2011). Kainic acid-induced neurotoxicity: targeting glial responses and glia-derived cytokines. Curr. Neuropharmacol. 9, 388-398. doi: 10.2174/157015911795596540

Zhou, J. N., Liu, R. Y., Kamphorst, W., Hofman, M. A., and Swaab, D. F. (2003). Early neuropathological Alzheimer's changes in aged individuals are accompanied by decreased cerebrospinal fluid melatonin levels. J. Pineal Res. 35, 125-130. doi: 10.1034/j.1600-079X.2003.00065.x

Conflict of Interest Statement: The authors declare that the research was conducted in the absence of any commercial or financial relationships that could be construed as a potential conflict of interest.

Copyright $\odot 2018$ Shi, Zeng, Li, Chen, Hang, Xia, Wu, Chen and Shi. This is an open-access article distributed under the terms of the Creative Commons Attribution License (CC BY). The use, distribution or reproduction in other forums is permitted, provided the original author(s) or licensor are credited and that the original publication in this journal is cited, in accordance with accepted academic practice. No use, distribution or reproduction is permitted which does not comply with these terms. 